\title{
Study of Inclusive $B^{-}$and $\bar{B}^{0}$ Decays to Flavor-Tagged $D, D_{s}$ and $\Lambda_{c}^{+}$
}

B. Aubert, R. Barate, M. Bona, D. Boutigny, F. Couderc, Y. Karyotakis, J. P. Lees, V. Poireau, V. Tisserand, and A. Zghiche Laboratoire de Physique des Particules, F-74941 Annecy-le-Vieux, France

E. Grauges

Universitat de Barcelona Fac. Fisica. Dept. ECM Avda Diagonal 647, 6a planta E-08028 Barcelona, Spain

A. Palano and M. Pappagallo

Università di Bari, Dipartimento di Fisica and INFN, I-70126 Bari, Italy

J. C. Chen, N. D. Qi, G. Rong, P. Wang, and Y. S. Zhu Institute of High Energy Physics, Beijing 100039, China

G. Eigen, I. Ofte, and B. Stugu

University of Bergen, Institute of Physics, N-5007 Bergen, Norway

G. S. Abrams, M. Battaglia, D. N. Brown, J. Button-Shafer, R. N. Cahn, E. Charles, C. T. Day, M. S. Gill,

Y. Groysman, R. G. Jacobsen, J. A. Kadyk, L. T. Kerth, Yu. G. Kolomensky, G. Kukartsev, G. Lynch,

L. M. Mir, P. J. Oddone, T. J. Orimoto, M. Pripstein, N. A. Roe, M. T. Ronan, and W. A. Wenzel Lawrence Berkeley National Laboratory and University of California, Berkeley, California 94720, USA

M. Barrett, K. E. Ford, T. J. Harrison, A. J. Hart, C. M. Hawkes, S. E. Morgan, and A. T. Watson University of Birmingham, Birmingham, B15 2TT, United Kingdom

K. Goetzen, T. Held, H. Koch, B. Lewandowski, M. Pelizaeus, K. Peters, T. Schroeder, and M. Steinke Ruhr Universität Bochum, Institut für Experimentalphysik 1, D-44780 Bochum, Germany

J. T. Boyd, J. P. Burke, W. N. Cottingham, and D. Walker University of Bristol, Bristol BS8 1TL, United Kingdom

T. Cuhadar-Donszelmann, B. G. Fulsom, C. Hearty, N. S. Knecht, T. S. Mattison, and J. A. McKenna University of British Columbia, Vancouver, British Columbia, Canada V6T $1 Z 1$

A. Khan, P. Kyberd, M. Saleem, and L. Teodorescu Brunel University, Uxbridge, Middlesex UB8 3PH, United Kingdom

V. E. Blinov, A. D. Bukin, V. P. Druzhinin, V. B. Golubev, A. P. Onuchin, S. I. Serednyakov, Yu. I. Skovpen, E. P. Solodov, and K. Yu Todyshev Budker Institute of Nuclear Physics, Novosibirsk 630090, Russia

D. S. Best, M. Bondioli, M. Bruinsma, M. Chao, S. Curry, I. Eschrich, D. Kirkby, A. J. Lankford, P. Lund, M. Mandelkern, R. K. Mommsen, W. Roethel, and D. P. Stoker University of California at Irvine, Irvine, California 92697, USA

S. Abachi and C. Buchanan

University of California at Los Angeles, Los Angeles, California 90024, USA

S. D. Foulkes, J. W. Gary, O. Long, B. C. Shen, K. Wang, and L. Zhang

University of California at Riverside, Riverside, California 92521, USA

Submitted to Physical Review D

Work supported in part by Department of Energy contract DE-AC02-76SF00515 
H. K. Hadavand, E. J. Hill, H. P. Paar, S. Rahatlou, and V. Sharma

University of California at San Diego, La Jolla, California 92093, USA

J. W. Berryhill, C. Campagnari, A. Cunha, B. Dahmes, T. M. Hong, D. Kovalskyi, and J. D. Richman University of California at Santa Barbara, Santa Barbara, California 93106, USA

T. W. Beck, A. M. Eisner, C. J. Flacco, C. A. Heusch, J. Kroseberg, W. S. Lockman, G. Nesom,

T. Schalk, B. A. Schumm, A. Seiden, P. Spradlin, D. C. Williams, and M. G. Wilson

University of California at Santa Cruz, Institute for Particle Physics, Santa Cruz, California 95064, USA

J. Albert, E. Chen, A. Dvoretskii, D. G. Hitlin, I. Narsky, T. Piatenko, F. C. Porter, A. Ryd, and A. Samuel California Institute of Technology, Pasadena, California 91125, USA

R. Andreassen, G. Mancinelli, B. T. Meadows, and M. D. Sokoloff University of Cincinnati, Cincinnati, Ohio 45221, USA

F. Blanc, P. C. Bloom, S. Chen, W. T. Ford, J. F. Hirschauer, A. Kreisel, U. Nauenberg,

A. Olivas, W. O. Ruddick, J. G. Smith, K. A. Ulmer, S. R. Wagner, and J. Zhang University of Colorado, Boulder, Colorado 80309, USA

A. Chen, E. A. Eckhart, A. Soffer, W. H. Toki, R. J. Wilson, F. Winklmeier, and Q. Zeng Colorado State University, Fort Collins, Colorado 80523, USA

D. D. Altenburg, E. Feltresi, A. Hauke, H. Jasper, and B. Spaan Universität Dortmund, Institut für Physik, D-44221 Dortmund, Germany

T. Brandt, V. Klose, H. M. Lacker, W. F. Mader, R. Nogowski, A. Petzold,

J. Schubert, K. R. Schubert, R. Schwierz, J. E. Sundermann, and A. Volk

Technische Universität Dresden, Institut für Kern- und Teilchenphysik, D-01062 Dresden, Germany

D. Bernard, G. R. Bonneaud, P. Grenier, ${ }^{*}$ E. Latour, Ch. Thiebaux, and M. Verderi Ecole Polytechnique, LLR, F-91128 Palaiseau, France

D. J. Bard, P. J. Clark, W. Gradl, F. Muheim, S. Playfer, A. I. Robertson, and Y. Xie University of Edinburgh, Edinburgh EH9 3JZ, United Kingdom

M. Andreotti, D. Bettoni, C. Bozzi, R. Calabrese, G. Cibinetto,

E. Luppi, M. Negrini, A. Petrella, L. Piemontese, and E. Prencipe Università di Ferrara, Dipartimento di Fisica and INFN, I-44100 Ferrara, Italy

F. Anulli, R. Baldini-Ferroli, A. Calcaterra, R. de Sangro, G. Finocchiaro, S. Pacetti, P. Patteri, I. M. Peruzzi, ${ }^{\dagger}$ M. Piccolo, M. Rama, and A. Zallo Laboratori Nazionali di Frascati dell'INFN, I-00044 Frascati, Italy

A. Buzzo, R. Capra, R. Contri, M. Lo Vetere, M. M. Macri, M. R. Monge,

S. Passaggio, C. Patrignani, E. Robutti, A. Santroni, and S. Tosi

Università di Genova, Dipartimento di Fisica and INFN, I-16146 Genova, Italy

G. Brandenburg, K. S. Chaisanguanthum, M. Morii, and J. Wu

Harvard University, Cambridge, Massachusetts 02138, USA

R. S. Dubitzky, J. Marks, S. Schenk, and U. Uwer

Universität Heidelberg, Physikalisches Institut, Philosophenweg 12, D-69120 Heidelberg, Germany

W. Bhimji, D. A. Bowerman, P. D. Dauncey, U. Egede, R. L. Flack,

J. R. Gaillard, J .A. Nash, M. B. Nikolich, and W. Panduro Vazquez Imperial College London, London, SW7 2AZ, United Kingdom 
X. Chai, M. J. Charles, U. Mallik, N. T. Meyer, and V. Ziegler

University of Iowa, Iowa City, Iowa 52242, USA

J. Cochran, H. B. Crawley, L. Dong, V. Eyges, W. T. Meyer, S. Prell, E. I. Rosenberg, and A. E. Rubin Iowa State University, Ames, Iowa 50011-3160, USA

A. V. Gritsan

Johns Hopkins Univ. Dept of Physics \& Astronomy 3400 N. Charles Street Baltimore, Maryland 21218

M. Fritsch and G. Schott

Universität Karlsruhe, Institut für Experimentelle Kernphysik, D-76021 Karlsruhe, Germany

N. Arnaud, M. Davier, G. Grosdidier, A. Höcker, F. Le Diberder, V. Lepeltier, A. M. Lutz, A. Oyanguren,

S. Pruvot, S. Rodier, P. Roudeau, M. H. Schune, A. Stocchi, W. F. Wang, and G. Wormser

Laboratoire de l'Accélérateur Linéaire, IN2P3-CNRS et Université Paris-Sud 11,

Centre Scientifique d'Orsay, B.P. 34, F-91898 ORSAY Cedex, France

C. H. Cheng, D. J. Lange, and D. M. Wright

Lawrence Livermore National Laboratory, Livermore, California 94550, USA

C. A. Chavez, I. J. Forster, J. R. Fry, E. Gabathuler, R. Gamet, K. A. George,

D. E. Hutchcroft, D. J. Payne, K. C. Schofield, and C. Touramanis

University of Liverpool, Liverpool L69 7ZE, United Kingdom

A. J. Bevan, F. Di Lodovico, W. Menges, and R. Sacco

Queen Mary, University of London, E1 4NS, United Kingdom

C. L. Brown, G. Cowan, H. U. Flaecher, D. A. Hopkins, P. S. Jackson, T. R. McMahon, S. Ricciardi, and F. Salvatore

University of London, Royal Holloway and Bedford New College, Egham, Surrey TW20 OEX, United Kingdom

D. N. Brown and C. L. Davis

University of Louisville, Louisville, Kentucky 40292, USA

J. Allison, N. R. Barlow, R. J. Barlow, Y. M. Chia, C. L. Edgar,

M. P. Kelly, G. D. Lafferty, M. T. Naisbit, J. C. Williams, and J. I. Yi

University of Manchester, Manchester M13 9PL, United Kingdom

C. Chen, W. D. Hulsbergen, A. Jawahery, C. K. Lae, D. A. Roberts, and G. Simi

University of Maryland, College Park, Maryland 20742, USA

G. Blaylock, C. Dallapiccola, S. S. Hertzbach, X. Li, T. B. Moore, S. Saremi, H. Staengle, and S. Y. Willocq

University of Massachusetts, Amherst, Massachusetts 01003, USA

R. Cowan, K. Koeneke, G. Sciolla, S. J. Sekula, M. Spitznagel, F. Taylor, and R. K. Yamamoto Massachusetts Institute of Technology, Laboratory for Nuclear Science, Cambridge, Massachusetts 02139, USA

H. Kim, P. M. Patel, C. T. Potter, and S. H. Robertson

McGill University, Montréal, Québec, Canada H3A $2 T 8$

A. Lazzaro, V. Lombardo, and F. Palombo

Università di Milano, Dipartimento di Fisica and INFN, I-20133 Milano, Italy

J. M. Bauer, L. Cremaldi, V. Eschenburg, R. Godang, R. Kroeger,

J. Reidy, D. A. Sanders, D. J. Summers, and H. W. Zhao

University of Mississippi, University, Mississippi 38677, USA

S. Brunet, D. Côté, M. Simard, P. Taras, and F. B. Viaud

Université de Montréal, Physique des Particules, Montréal, Québec, Canada HЗC 3Jł 


\author{
H. Nicholson \\ Mount Holyoke College, South Hadley, Massachusetts 01075, USA
}

N. Cavallo $\ddagger$ G. De Nardo, D. del Re, F. Fabozzi $₫$ C. Gatto, L. Lista, D. Monorchio, D. Piccolo, and C. Sciacca Università di Napoli Federico II, Dipartimento di Scienze Fisiche and INFN, I-80126, Napoli, Italy

M. Baak, H. Bulten, G. Raven, and H. L. Snoek

NIKHEF, National Institute for Nuclear Physics and High Energy Physics, NL-1009 DB Amsterdam, The Netherlands

C. P. Jessop and J. M. LoSecco

University of Notre Dame, Notre Dame, Indiana 46556, USA

T. Allmendinger, G. Benelli, K. K. Gan, K. Honscheid, D. Hufnagel, P. D. Jackson,

H. Kagan, R. Kass, T. Pulliam, A. M. Rahimi, R. Ter-Antonyan, and Q. K. Wong Ohio State University, Columbus, Ohio 43210, USA

N. L. Blount, J. Brau, R. Frey, O. Igonkina, M. Lu, R. Rahmat, N. B. Sinev, D. Strom, J. Strube, and E. Torrence University of Oregon, Eugene, Oregon 97403, USA

F. Galeazzi, A. Gaz, M. Margoni, M. Morandin, A. Pompili,

M. Posocco, M. Rotondo, F. Simonetto, R. Stroili, and C. Voci Università di Padova, Dipartimento di Fisica and INFN, I-35131 Padova, Italy

M. Benayoun, J. Chauveau, P. David, L. Del Buono, Ch. de la Vaissière, O. Hamon,

B. L. Hartfiel, M. J. J. John, Ph. Leruste, J. Malclès, J. Ocariz, L. Roos, and G. Therin

Universités Paris VI et VII, Laboratoire de Physique Nucléaire et de Hautes Energies, F-75252 Paris, France

P. K. Behera, L. Gladney, and J. Panetta

University of Pennsylvania, Philadelphia, Pennsylvania 19104, USA

M. Biasini, R. Covarelli, and M. Pioppi

Università di Perugia, Dipartimento di Fisica and INFN, I-06100 Perugia, Italy

C. Angelini, G. Batignani, S. Bettarini, F. Bucci, G. Calderini, M. Carpinelli, R. Cenci, F. Forti, M. A. Giorgi,

A. Lusiani, G. Marchiori, M. A. Mazur, M. Morganti, N. Neri, E. Paoloni, G. Rizzo, and J. Walsh

Università di Pisa, Dipartimento di Fisica, Scuola Normale Superiore and INFN, I-56127 Pisa, Italy

M. Haire, D. Judd, and D. E. Wagoner

Prairie View A\&M University, Prairie View, Texas 77446, USA

J. Biesiada, N. Danielson, P. Elmer, Y. P. Lau, C. Lu, J. Olsen, A. J. S. Smith, and A. V. Telnov Princeton University, Princeton, New Jersey 08544, USA

F. Bellini, G. Cavoto, A. D’Orazio, E. Di Marco, R. Faccini, F. Ferrarotto, F. Ferroni, M. Gaspero, L. Li Gioi, M. A. Mazzoni, S. Morganti, G. Piredda, F. Polci, F. Safai Tehrani, and C. Voena Università di Roma La Sapienza, Dipartimento di Fisica and INFN, I-00185 Roma, Italy

M. Ebert, H. Schröder, and R. Waldi Universität Rostock, D-18051 Rostock, Germany

T. Adye, N. De Groot, B. Franek, E. O. Olaiya, and F. F. Wilson Rutherford Appleton Laboratory, Chilton, Didcot, Oxon, OX11 0QX, United Kingdom

S. Emery, A. Gaidot, S. F. Ganzhur, G. Hamel de Monchenault, W. Kozanecki, M. Legendre, B. Mayer, G. Vasseur, Ch. Yèche, and M. Zito DSM/Dapnia, CEA/Saclay, F-91191 Gif-sur-Yvette, France

W. Park, M. V. Purohit, A. W. Weidemann, and J. R. Wilson 
University of South Carolina, Columbia, South Carolina 29208, USA

M. T. Allen, D. Aston, R. Bartoldus, P. Bechtle, N. Berger, A. M. Boyarski, R. Claus, J. P. Coleman, M. R. Convery, M. Cristinziani, J. C. Dingfelder, D. Dong, J. Dorfan, G. P. Dubois-Felsmann, D. Dujmic, W. Dunwoodie, R. C. Field, T. Glanzman, S. J. Gowdy, M. T. Graham, V. Halyo, C. Hast, T. Hryn'ova, W. R. Innes, M. H. Kelsey, P. Kim, M. L. Kocian, D. W. G. S. Leith, S. Li, J. Libby, S. Luitz, V. Luth, H. L. Lynch, D. B. MacFarlane, H. Marsiske, R. Messner, D. R. Muller, C. P. O'Grady, V. E. Ozcan, A. Perazzo, M. Perl, B. N. Ratcliff, A. Roodman, A. A. Salnikov, R. H. Schindler, J. Schwiening, A. Snyder, J. Stelzer, D. Su, M. K. Sullivan, K. Suzuki, S. K. Swain, J. M. Thompson, J. Va'vra, N. van Bakel, M. Weaver, A. J. R. Weinstein, W. J. Wisniewski, M. Wittgen, D. H. Wright, A. K. Yarritu, K. Yi, and C. C. Young Stanford Linear Accelerator Center, Stanford, California 94309, USA

P. R. Burchat, A. J. Edwards, S. A. Majewski, B. A. Petersen, C. Roat, and L. Wilden Stanford University, Stanford, California 94305-4060, USA

S. Ahmed, M. S. Alam, R. Bula, J. A. Ernst, V. Jain, B. Pan, M. A. Saeed, F. R. Wappler, and S. B. Zain State University of New York, Albany, New York 12222, USA

W. Bugg, M. Krishnamurthy, and S. M. Spanier University of Tennessee, Knoxville, Tennessee 37996, USA

R. Eckmann, J. L. Ritchie, A. Satpathy, C. J. Schilling, and R. F. Schwitters University of Texas at Austin, Austin, Texas 78712, USA

J. M. Izen, I. Kitayama, X. C. Lou, and S. Ye University of Texas at Dallas, Richardson, Texas 75083, USA

F. Bianchi, F. Gallo, and D. Gamba

Università di Torino, Dipartimento di Fisica Sperimentale and INFN, I-10125 Torino, Italy

M. Bomben, L. Bosisio, C. Cartaro, F. Cossutti, G. Della Ricca, S. Dittongo, S. Grancagnolo, L. Lanceri, and L. Vitale Università di Trieste, Dipartimento di Fisica and INFN, I-34127 Trieste, Italy

V. Azzolini and F. Martinez-Vidal

IFIC, Universitat de Valencia-CSIC, E-46071 Valencia, Spain

Sw. Banerjee, B. Bhuyan, C. M. Brown, D. Fortin, K. Hamano,

R. Kowalewski, I. M. Nugent, J. M. Roney, and R. J. Sobie

University of Victoria, Victoria, British Columbia, Canada V8W 3P6

J. J. Back, P. F. Harrison, T. E. Latham, and G. B. Mohanty

Department of Physics, University of Warwick, Coventry CV4 7AL, United Kingdom

H. R. Band, X. Chen, B. Cheng, S. Dasu, M. Datta, A. M. Eichenbaum, K. T. Flood, J. J. Hollar, J. R. Johnson, P. E. Kutter, H. Li, R. Liu, B. Mellado, A. Mihalyi, A. K. Mohapatra, Y. Pan, M. Pierini, R. Prepost, P. Tan, S. L. Wu, and Z. Yu University of Wisconsin, Madison, Wisconsin 53706, USA

H. Neal

Yale University, New Haven, Connecticut 06511, USA

(Dated: June 12, 2006)

We report on a study of inclusive $B^{-}$and $\bar{B}^{0}$ meson decays to $D^{0} X, \bar{D}^{0} X, D^{+} X, D^{-} X, D_{s}^{+} X$, $D_{s}^{-} X, \Lambda_{c}^{+} X, \bar{\Lambda}_{c}^{-} X$, based on a sample of 231 million $B \bar{B}$ events recorded with the BABAR detector at the $\Upsilon(4 S)$ resonance. Events are selected by completely reconstructing one $B$ and searching for a reconstructed charm particle in the rest of the event. From the measured branching fractions of these decays, we infer the number of charm and anti-charm particles per $\bar{B}$ decay, separately for charged and neutral parents. We derive the total charm yield per $B^{-}$decay, $n_{c}^{-}=1.202 \pm 0.023 \pm 0.040_{-0.029}^{+0.035}$, 
and per $\bar{B}^{0}$ decay, $n_{c}^{0}=1.193 \pm 0.030 \pm 0.034_{-0.035}^{+0.044}$ where the first uncertainty is statistical, the second is systematic, and the third reflects the charm branching-fraction uncertainties. We also present the charm momentum distributions measured in the $\bar{B}$ rest frame.

PACS numbers: 13.25.Hw, 12.15.Hh, 11.30.Er

\section{INTRODUCTION}

The dominant process for the decay of a $b$ quark is $b \rightarrow c W^{*-}[1]$, resulting in a (flavor) correlated $c$ quark and a virtual $W$. In the decay of the $W$, the production of a $\bar{u} d$ or a $\bar{c} s$ pair are both Cabibbo-allowed and should be approximately equal, the latter being suppressed by a phase-space factor. The first process dominates hadronic $b$ decays. The second can be easily distinguished as it produces a (flavor) anticorrelated $\bar{c}$ quark. Experimentally, we investigate correlated and anticorrelated charm production through the measurement of the inclusive $B$ decay rates to a limited number of charm hadron species, i.e. $D^{0}, \bar{D}^{0}, D^{+}, D^{-}, D_{s}^{+}, D_{s}^{-}, \Lambda_{c}^{+}, \bar{\Lambda}_{c}^{-}, \Xi_{c}$ and charmonia, because all other charm particles decay into one of the previous hadrons.

The analysis presented here exploits a substantially larger data sample than the original $B A B A R$ result [2]. It also employs a more sophisticated fitting method to extract, in a correlated manner, the number of reconstructed $B$ mesons and the charm hadron yields, which reduces the experimental systematic uncertainty. Other measurements [3-7] of these rates are more statistically limited and/or do not distinguish between the different parent $B$ states. Besides the theoretical interest [8-11], the fact that anticorrelated charm particles are a background for many studies also motivates a more precise measurement of their production rates in $B$ decays.

Most of the charged and neutral $D$ mesons produced in $\bar{B}$ decays come from correlated production $\bar{B} \rightarrow D X$. However, a significant number of $\bar{B} \rightarrow \bar{D} X$ decays are expected through $b \rightarrow c \bar{c} s$ transitions, such as $\bar{B} \rightarrow$ $D^{(*)} \bar{D}^{(*)} \bar{K}^{(*)}(n \pi)$. Although the branching fractions of the 3-body decays $\bar{B} \rightarrow D^{(*)} \bar{D}^{(*)} \bar{K}$ have been measured $[12,13]$, they do not saturate $\bar{B} \rightarrow \bar{D} X$ transitions [2]. It is therefore important to improve the precision on the $\bar{B} \rightarrow \bar{D} X$ branching fraction.

By contrast, anticorrelated $D_{s}^{-}$production, $\bar{B} \rightarrow D_{s}^{-} D(n \pi)$, is expected to dominate $\bar{B}$ decays to $D_{s}$ mesons, since correlated production needs an extra $s \bar{s}$ pair created from the vacuum to give $\bar{B} \rightarrow D_{s}^{+} K^{-}(n \pi)$. There is no prior published measurement for correlated $D_{s}^{+}$production.

\footnotetext{
*Also at Laboratoire de Physique Corpusculaire, ClermontFerrand, France

${ }^{\dagger}$ Also with Università di Perugia, Dipartimento di Fisica, Perugia, Italy

¥Also with Università della Basilicata, Potenza, Italy
}

Correlated $\Lambda_{c}^{+}$are produced in decays like $\bar{B} \rightarrow \Lambda_{c}^{+} \bar{p} \pi^{-}(\pi)$, while anticorrelated $\bar{\Lambda}_{c}^{-}$should originate predominantly from $\bar{B} \rightarrow \Xi_{c} \bar{\Lambda}_{c}^{-}(\pi)$. The decay $\bar{B} \rightarrow \Xi_{c} \bar{\Lambda}_{c}^{-}$has recently been observed [14], confirming the hypothesis of associated $\Xi_{c} \bar{\Lambda}_{c}^{-}$production. Another possibility for anticorrelated $\bar{\Lambda}_{c}^{-}$production is $\bar{B} \rightarrow \Lambda_{c}^{+} \bar{\Lambda}_{c}^{-} K$, the baryonic analogue of the $D \bar{D} K$ decay.

This analysis uses $\Upsilon(4 S) \rightarrow B \bar{B}$ events in which either a $B^{+}$or a $B^{0}$ meson (hereafter denoted $B_{\text {rec }^{\prime} \mathrm{d}}$ ) decays into a hadronic final state and is fully reconstructed. We then reconstruct $D, D_{s}$ and $\Lambda_{c}^{+}$from the decay products of the recoiling $B^{-}\left(\bar{B}^{0}\right)$ meson and compare the flavor of the charm hadron with that of the reconstructed $B$ (taking into account $B^{0}-\bar{B}^{0}$ mixing). This allows separate measurements of the $B^{-}\left(\bar{B}^{0}\right) \rightarrow D^{0} X, D^{+} X, D_{s}^{+}$ $X, \Lambda_{c}^{+} X$ and $B^{-}\left(\bar{B}^{0}\right) \rightarrow \bar{D}^{0} X, D^{-} X, D_{s}^{-} X, \bar{\Lambda}_{c}^{-} X$ branching fractions.

We then compute the average number of correlated (anticorrelated) charm particles per $B^{-}$decay, $N_{c}^{-}$ $\left(N_{\bar{c}}^{-}\right):$

$$
\begin{aligned}
& N_{c}^{-}=\sum_{C} \mathcal{B}\left(B^{-} \rightarrow C X\right), \\
& N_{\bar{c}}^{-}=\sum_{\bar{C}} \mathcal{B}\left(B^{-} \rightarrow \bar{C} X\right),
\end{aligned}
$$

where the sum is performed over $C \equiv$ $\left\{D^{0}, \quad D^{+}, \quad D_{s}^{+}, \quad \Lambda_{c}^{+}, \quad \Xi_{c}, \quad(c \bar{c})\right\} \quad$ or $\quad \bar{C} \equiv$ $\left\{\bar{D}^{0}, D^{-}, D_{s}^{-}, \bar{\Lambda}_{c}^{-},(c \bar{c})\right\}$, where $(c \bar{c})$ refers to all charmonium states collectively. We neglect anticorrelated $\bar{\Xi}_{c}$ production, as it requires both a $\bar{c} s$ and an $s \bar{s}$ pair in the decay to give $\bar{\Xi}_{c} \Omega_{c}$. We then sum $N_{c}^{-}$ and $N_{\bar{c}}^{-}$to obtain the average number of charm plus anti-charm quarks per $B^{-}$decay, $n_{c}^{-}=N_{c}^{-}+N_{\bar{c}}^{-}$. We similarly define $N_{c}^{0}, N_{\bar{c}}^{0}$ and $n_{c}^{0}$ for $\bar{B}^{0}$ decays.

The above method also lends itself to a measurement of the momentum distribution of each charm species directly in the rest frame of the parent meson, because the four-momentum of each recoiling $\bar{B}$ is fully determined from those of the $\Upsilon(4 S)$ and of the reconstructed $B$. The resulting charm spectra can then be compared to theoretical predictions in the same frame [15]. This avoids the significant smearing due to the Lorentz boost from the parent- $\bar{B}$ frame to the $\Upsilon(4 S)$ frame affecting earlier measurements, such as those reported in [3]. These spectra might also show indications of four-quark states [16]. 


\section{BABAR DETECTOR AND DATA SAMPLE}

The measurements presented here are based on a sample of 231 million $B \bar{B}$ pairs $\left(210 \mathrm{fb}^{-1}\right)$ recorded at the $\Upsilon(4 S)$ resonance with the BABAR detector at the PEPII asymmetric-energy $B$ factory at SLAC. The BABAR detector is described in detail elsewhere [17]. Chargedparticle trajectories are measured by a 5-layer doublesided silicon vertex tracker and a 40-layer drift chamber, both operating in a 1.5-T solenoidal magnetic field. Charged-particle identification is provided by the average energy loss $(\mathrm{d} E / \mathrm{d} x)$ in the tracking devices and by an internally reflecting ring-imaging Cherenkov detector. Photons are detected by a CsI(Tl) electromagnetic calorimeter. We use Monte Carlo simulations of the BABAR detector based on GEANT4 [18] to optimize selection criteria and determine selection efficiencies.

\section{III. $B$ MESON RECONSTRUCTION}

We reconstruct $B^{+}$and $B^{0}$ decays $\left(B_{\text {rec }^{\prime} \mathrm{d}}\right)$ in the modes $B^{+} \rightarrow \bar{D}^{(*) 0} \pi^{+}, \bar{D}^{(*) 0} \rho^{+}, \bar{D}^{(*) 0} a_{1}^{+}$and $B^{0} \rightarrow$ $D^{(*)-} \pi^{+}, D^{(*)-} \rho^{+}, D^{(*)-} a_{1}^{+} . \quad \bar{D}^{0}$ candidates are reconstructed in the $K^{+} \pi^{-}, K^{+} \pi^{-} \pi^{0}, K^{+} \pi^{-} \pi^{+} \pi^{-}$and $K_{S}^{0} \pi^{+} \pi^{-}\left(K_{S}^{0} \rightarrow \pi^{+} \pi^{-}\right)$decay channels, while $D^{-}$are reconstructed in the $K^{+} \pi^{-} \pi^{-}$and $K_{S}^{0} \pi^{-}$modes. $D^{*}$ candidates are reconstructed in the $D^{*-} \rightarrow \bar{D}^{0} \pi^{-}$and $\bar{D}^{* 0} \rightarrow \bar{D}^{0} \pi^{0}$ decay modes.

The kinematic selection of fully reconstructed $B$ decays relies on two variables. The first is $\Delta E=E_{B}^{*}-\sqrt{s} / 2$, where $E_{B}^{*}$ is the energy of the reconstructed $B$ candidate in the $e^{+} e^{-}$center-of-mass frame and $\sqrt{s}$ is the invariant mass of the initial $e^{+} e^{-}$system. The second is the beam-energy substituted mass, defined by $m_{\mathrm{ES}}=\sqrt{\left(s / 2+\mathbf{p}_{i} \cdot \mathbf{p}_{B}\right)^{2} / E_{i}^{2}-\mathbf{p}_{B}^{2}}$, where $\mathbf{p}_{B}$ is the $B_{\text {rec'd }}$ momentum and $\left(E_{i}, \mathbf{p}_{i}\right)$ is the four-momentum of the initial $e^{+} e^{-}$system, both measured in the laboratory frame. We require $|\Delta E|<n \sigma_{\Delta E}$, using the resolution $\sigma_{\Delta E}$ measured for each decay mode, with $n=2$ or 3 depending on the decay mode. If an event contains several $B^{+}\left(B^{0}\right)$ candidates, only the highest-purity $B$-decay mode is retained. The purity is defined, for each $B$-decay mode separately, as the fraction of signal $B$ decays with $m_{\mathrm{ES}}>5.27 \mathrm{GeV} / c^{2}$, normalized to the total number of reconstructed $B^{+}\left(B^{0}\right)$ candidates in same interval.

The signal yield $N_{B}$ of reconstructed $B$ mesons is extracted from a fit to the $m_{\mathrm{ES}}$ spectra (Fig. 1). The $B$ signal is modeled by a Crystal Ball signal function $\Gamma_{C B}[19]$ which is a Gaussian peaking at the $B$ meson mass modified by an exponential low-mass tail that accounts for photon energy loss. The $B$ combinatorial background is modeled using the empirical ARGUS phase-space threshold function $\Gamma_{A R G}[20]$. All the signal and background parameters in these functions are extracted from the data. The signal yields of reconstructed $B^{+}$and $B^{0}$ mesons are $N_{B^{+}}=200359 \pm 705$ and $N_{B^{0}}=110735 \pm 424$, where the errors reflect the statistical uncertainty in the


FIG. 1: $m_{\text {ES }}$ spectra of reconstructed (a) $B^{+}$and (b) $B^{0}$ candidates. The solid curve is the sum of the fitted signal and background whereas the dashed curve is the background component only.

number of combinatorial background events. These numbers provide the normalization for all the branching fractions reported below.

The contamination of misreconstructed $B^{0}$ events in the $B^{+}$signal (and vice-versa) induces a background which peaks near the $B$ mass. From the Monte Carlo simulation, the fraction of $B^{0}$ events in the reconstructed $B^{+}$signal sample is found to be $c_{0}=0.038 \pm 0.009$ (syst), and the fraction of $B^{+}$events in the reconstructed $B^{0}$ signal sample $c_{+}=0.028 \pm 0.007$ (syst). The systematic uncertainties take into account possible differences in reconstructing real or simulated events, as well as branching-fraction uncertainties for those $B$ decay modes contributing to the wrong-charge contamination.

\section{INCLUSIVE CHARM BRANCHING FRACTIONS}

We now turn to the analysis of inclusive $D, \bar{D}, D_{s}^{-}$, $D_{s}^{+}, \Lambda_{c}^{+}$and $\bar{\Lambda}_{c}^{-}$production in the decays of the $\bar{B}$ mesons that recoil against the reconstructed $B$. Charm particles $C$ are distinguished from anti-charm particles $\bar{C}$. They 



FIG. 2: Charm (left) and anti-charm (right) mass spectra in the recoil of $B^{+}$candidates, for the subsample of events with $m_{\mathrm{ES}}>5.270 \mathrm{GeV} / c^{2}$ ( $B$ signal region). The solid curve shows the result of the two-dimensional fit. The dark shaded areas show the contribution of reconstructed $D, \bar{D}, \Lambda_{c}^{+}$and $\bar{\Lambda}_{c}^{-}$signal in the recoil of combinatorial $B_{\mathrm{rec}^{\prime} \mathrm{d}}^{+}$background. The light shaded area corresponds to the fitted combinatorial (anti-) charm background.

are reconstructed from charged tracks that do not belong to the reconstructed $B$. The decay modes considered are listed in Table I along with their branching fractions. Those are taken from Ref. [21] except in the case of the $D_{s}^{+} \rightarrow \phi \pi^{+}$channel [22] for which we use the more precise measurement reported in Ref. [23].
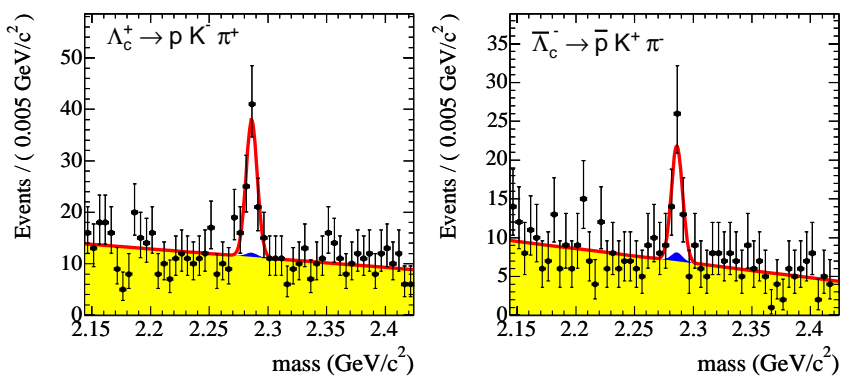

FIG. 3: Charm (left) and anti-charm (right) mass spectra as for Fig. 2 but in the recoil of $\bar{B}^{0}$ candidates.

\section{A. Charm particle yields}

The numbers of charm (anti-charm) particles are extracted from an unbinned maximum likelihood fit to the two-dimensional distribution $\left[m_{\mathrm{ES}}, m_{C(\bar{C})}\right]$, where $m_{\mathrm{ES}}$ is the beam-energy substituted mass of the reconstructed $B$ and $m_{C(\bar{C})}$ is the mass of the charm (anti-charm) particle found among the recoil products. Figs. 2 to 5 show the results of these fits, projected onto the $m_{C(\bar{C})}$ axis, for events in the $m_{\mathrm{ES}}$ signal region $\left(m_{\mathrm{ES}}>5.270 \mathrm{GeV} / c^{2}\right)$. The probability density function used to fit the $\left[m_{\mathrm{ES}}, m_{C(\bar{C})}\right]$ distributions is the sum of four components : 

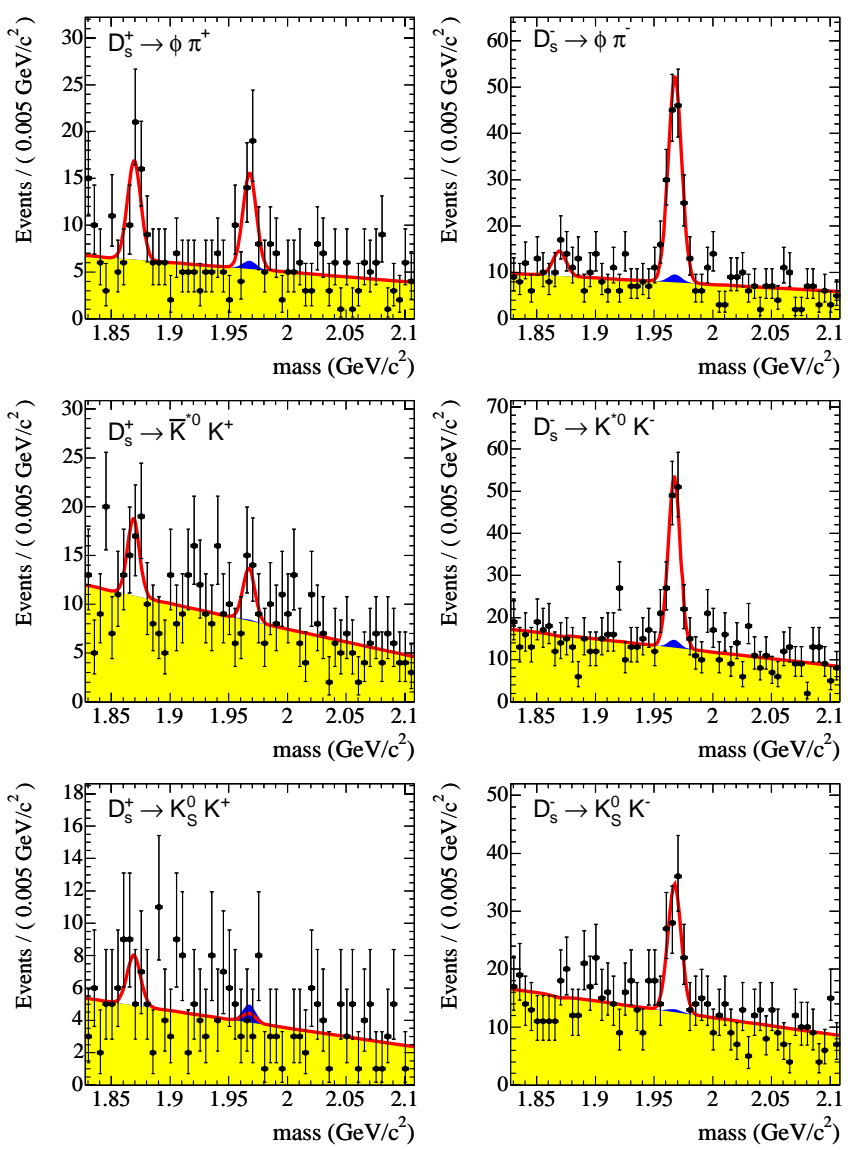

FIG. 4: $D_{s}^{+}$(left) and $D_{s}^{-}$(right) mass spectra in the recoil of $B^{+}$candidates, for the subsample of events with $m_{\mathrm{ES}}>5.270 \mathrm{GeV} / c^{2}$ ( $B$ signal region). The solid curve shows the result of the two-dimensional fit. The dark shaded areas show the contribution of reconstructed $D_{s}^{+}, D_{s}^{-}$signal in the recoil of combinatorial $B_{\mathrm{rec}^{\prime} \mathrm{d}}^{+}$background. The light shaded area corresponds to the fitted combinatorial (anti-) charm background. The Gaussian peak at the $D^{+}$mass accounts for reconstructed $D^{+}$signal [24].

- $P_{B s i g}^{C s i g}$ : reconstructed charm (anti-charm) signal in the recoil of reconstructed $B$ signal,

- $P_{B b k g}^{C s i g}$ : reconstructed charm (anti-charm) signal in the recoil of combinatorial $B$ background,

- $P_{B s i g}^{C b k g}$ : combinatorial charm (anti-charm) background in the recoil of reconstructed $B$ signal,

- $P_{B b k g}^{C b k g}$ : combinatorial charm (anti-charm) background in the recoil of combinatorial $B$ background,
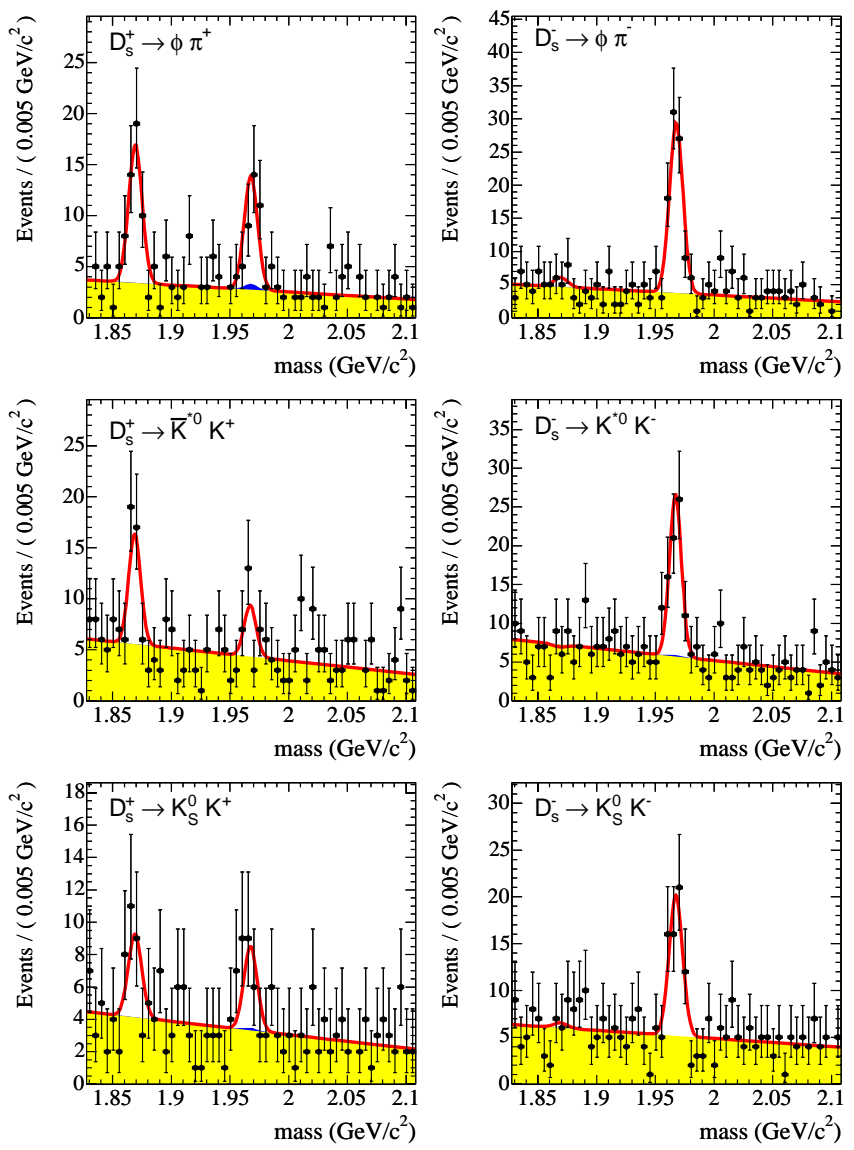

FIG. 5: $D_{s}^{+}$and $D_{s}^{-}$mass spectra as for Fig. 4 but in the recoil of $\bar{B}^{0}$ candidates.

These four components are modeled as follows :

$$
\begin{aligned}
& P_{B s i g}^{C s i g}\left(m_{\mathrm{ES}}, m_{C}\right) \equiv \Gamma_{C B}\left(m_{\mathrm{ES}}\right) \times \rho_{S}\left(m_{C}\right), \\
& P_{B b k g}^{C s i g}\left(m_{\mathrm{ES}}, m_{C}\right) \equiv \Gamma_{A R G}\left(m_{\mathrm{ES}} \times \rho_{S}\left(m_{C}\right),\right. \\
& P_{B s i g}^{C b k g}\left(m_{\mathrm{ES}}, m_{C}\right) \equiv \Gamma_{C B}\left(m_{\mathrm{ES}}\right) \times \rho_{c o m b}\left(m_{C}\right), \\
& P_{B b k g}^{C b k g}\left(m_{\mathrm{ES}}, m_{C}\right) \equiv \Gamma_{A R G}\left(m_{\mathrm{ES}}\right) \times \rho_{c o m b}\left(m_{C}\right) .
\end{aligned}
$$

TABLE I: Charm particle decay modes and branching fractions.

\begin{tabular}{ll}
\hline \hline$C \rightarrow f$ & $\mathcal{B}(C \rightarrow f)(\%)$ \\
\hline$D^{0} \rightarrow K^{-} \pi^{+}$ & $3.80 \pm 0.09$ \\
$D^{0} \rightarrow K^{-} \pi^{+} \pi^{-} \pi^{+}$ & $7.48 \pm 0.31$ \\
$D^{+} \rightarrow K^{-} \pi^{+} \pi^{+}$ & $9.1 \pm 0.7$ \\
$D_{s}^{+} \rightarrow \phi \pi^{+}\left(\phi \rightarrow K^{+} K^{-}\right)$ & $4.81 \pm 0.64(49.3 \pm 1.0 \%)$ \\
$D_{s}^{+} \rightarrow \bar{K}^{* 0} K^{+}\left(\bar{K}^{* 0} \rightarrow K^{-} \pi^{+}\right)$ & $4.57 \pm 0.69(66.51 \pm 0.01 \%)$ \\
$D_{s}^{+} \rightarrow K_{S}^{0} K^{+}\left(K_{S}^{0} \rightarrow \pi^{+} \pi^{-}\right)$ & $2.43 \pm 0.42(68.95 \pm 0.14 \%)$ \\
$\Lambda_{c}^{+} \rightarrow p K^{-} \pi^{+}$ & $5.0 \pm 1.3$ \\
\hline \hline
\end{tabular}


The function $\Gamma_{C B}$ with all its parameters fixed from the fit detailed in Sec. III is used to model the reconstructed $B$ signal. The combinatorial $B$ background is described as in Sec. III by an ARGUS function $\Gamma_{A R G}$ whose shape parameter is floated in the fit to allow for a possible charm decay-mode dependence of this background. A Gaussian function $\rho_{S}\left(m_{C(\bar{C})}\right)$ describes the mass shape of the reconstructed charm signal. Its mean is taken from the data. Its resolution, as measured in the data, is consistent with that in the simulation and is fixed. The combinatorial charm-background distribution is fitted with a linear function $\rho_{c o m b}\left(m_{C(\bar{C})}\right)$ (except for the $D^{0} \rightarrow K^{-} \pi^{+} \pi^{-} \pi^{+}$for which a quadratic dependence is assumed) [24].

TABLE II: $p^{*}$-averaged reconstruction efficiencies $\epsilon_{C}$ for each charm final state. The errors reflect the limited Monte Carlo statistics.

\begin{tabular}{lr}
\hline \hline$C \rightarrow f$ & $\epsilon_{C}(\%)$ \\
\hline$D^{0} \rightarrow K^{-} \pi^{+}$ & $50.2 \pm 0.3$ \\
$D^{0} \rightarrow K^{-}{ }^{+} \pi^{-} \pi^{+}$ & $20.1 \pm 0.2$ \\
$D^{+} \rightarrow K^{-}{ }^{+}{ }^{+} \pi^{+}$ & $33.7 \pm 0.2$ \\
$D_{s}^{+} \rightarrow \phi \pi^{+}$ & $33.0 \pm 0.8$ \\
$D_{s}^{+} \rightarrow \bar{K}^{* 0} K^{+}$ & $18.0 \pm 0.5$ \\
$D_{s}^{+} \rightarrow K_{S}^{0} K^{+}$ & $31.1 \pm 0.8$ \\
$\Lambda_{c}^{+} \rightarrow p K^{-} \pi^{+}$ & $26.7 \pm 0.9$ \\
\hline \hline
\end{tabular}

The reconstruction efficiencies for each charm final state $C \rightarrow f$ (Table II) are computed from the simulation as a function of $p^{*}$, the charm-particle momentum in the $\bar{B}$ rest frame, and applied event-by-event to obtain the efficiency-corrected charm and anti-charm signal yields. These are denoted respectively by $N^{-}(C \rightarrow f)$ $\left(N^{0}(C \rightarrow f)\right)$ and $N^{-}(\bar{C} \rightarrow \bar{f})\left(N^{0}(\bar{C} \rightarrow \bar{f})\right)$ and are listed in Table III. We then determine the charm and anti-charm fractional production rates $\mathcal{B}_{c}^{-(0)}$ and $\overline{\mathcal{B}}_{c}^{-(0)}$, defined as :

$$
\begin{aligned}
\mathcal{B}_{c}^{-(0)} & =N^{-(0)}(C \rightarrow f) /\left[N_{B^{+}\left(B^{0}\right)} \times \mathcal{B}(C \rightarrow f)\right], \\
\overline{\mathcal{B}}_{c}^{-(0)} & =N^{-(0)}(\bar{C} \rightarrow \bar{f}) /\left[N_{B^{+}\left(B^{0}\right)} \times \mathcal{B}(C \rightarrow f)\right],
\end{aligned}
$$

where $N_{B^{+}}\left(N_{B^{0}}\right)$ is the number of reconstructed $B^{+}$ $\left(B^{0}\right)$ mesons, and $\mathcal{B}(C \rightarrow f)$ is the $C \rightarrow f$ branching fraction reported in Table I. $\mathcal{B}_{c}^{-}, \overline{\mathcal{B}}_{c}^{-}, \mathcal{B}_{c}^{0}$ and $\overline{\mathcal{B}}_{c}^{0}$ are listed in Table III.

\section{B. Correlated and anticorrelated charm branching fractions}

For charged $B$, the branching fractions for correlated and anticorrelated $C$ production are given by :

$$
\begin{aligned}
\mathcal{B}\left(B^{-} \rightarrow C X\right) & =\mathcal{B}_{c}^{-}-c_{0} \mathcal{B}_{1}^{0}, \\
\mathcal{B}\left(B^{-} \rightarrow \bar{C} X\right) & =\overline{\mathcal{B}}_{c}^{-}-c_{0} \mathcal{B}_{2}^{0} .
\end{aligned}
$$

The correlated (anticorrelated) $B^{-} \rightarrow C X$ branching fraction is equal to the charm (anti-charm) fractional production rate $\mathcal{B}_{c}^{-}\left(\overline{\mathcal{B}}_{c}^{-}\right)$in the recoil of reconstructed $B^{+}$mesons modified by a small correction term $c_{0} \mathcal{B}_{1}^{0}$ $\left(c_{0} \mathcal{B}_{2}^{0}\right)$ that accounts for the $B^{0}$ contamination in the reconstructed $B^{+}$sample. The factors $\mathcal{B}_{1}^{0}$ and $\mathcal{B}_{2}^{0}$ depend on the measured $\bar{B}^{0} \rightarrow C X$ and $B^{0} \rightarrow C X$ branching fractions, and on the $B^{0} \bar{B}^{0}$ mixing parameter $\chi_{d}$ [21]. Doubly Cabibbo-suppressed $D^{0}$ decays $\left(D^{0} \rightarrow K^{+} \pi^{-}\right.$ and $D^{0} \rightarrow K^{+} \pi^{+} \pi^{-} \pi^{-}$) are also taken into account. We combine the results from the different $D^{0}$ and $D_{s}$ decay modes to extract the final branching fractions listed in Table IV. The probability of the correlated $D_{s}^{+}$production observed in $B^{-}$decays to be due to a background fluctuation is less than $5 \times 10^{-4}$.

For neutral $B$, charm and anti-charm production in the recoil of reconstructed $B^{0}$ mesons have to be corrected for $B^{0} \bar{B}^{0}$ mixing to obtain the correlated and anticorrelated charm branching fractions :

$$
\begin{aligned}
& \mathcal{B}\left(\bar{B}^{0} \rightarrow C X\right)=\frac{\mathcal{B}_{c}^{0}-\chi_{d}\left(\mathcal{B}_{c}^{0}+\overline{\mathcal{B}}_{c}^{0}\right)}{1-2 \chi_{d}}-c_{+} \mathcal{B}_{1}^{+}, \\
& \mathcal{B}\left(\bar{B}^{0} \rightarrow \bar{C} X\right)=\frac{\overline{\mathcal{B}}_{c}^{0}-\chi_{d}\left(\overline{\mathcal{B}}_{c}^{0}+\mathcal{B}_{c}^{0}\right)}{1-2 \chi_{d}}-c_{+} \mathcal{B}_{2}^{+} .
\end{aligned}
$$

The correction factors $c_{+} \mathcal{B}_{1}^{+}$and $c_{+} \mathcal{B}_{2}^{+}$account for $B^{+}$contamination in the $B^{0}$ sample and depend on the $B^{-} \rightarrow C X$ and $B^{+} \rightarrow C X$ branching fractions. Combining the different $D^{0}$ and $D_{s}$ modes, we obtain the final branching fractions listed in Table IV.

We also compute the fraction of anticorrelated charm production in $\bar{B}$ decays :

$$
w(\bar{C})=\frac{\mathcal{B}(\bar{B} \rightarrow \bar{C} X)}{\mathcal{B}(\bar{B} \rightarrow C X)+\mathcal{B}(\bar{B} \rightarrow \bar{C} X)} .
$$

Here, many systematic uncertainties cancel out (tracking, $K$ identification, $D$ branching fractions, $B$ counting). The results are given in Table V.

The main systematic uncertainties are associated with the track-finding efficiency, the models used to describe the $m_{\mathrm{ES}}$ and $m_{C(\bar{C})}$ distributions, and the particle identification efficiency. For example, the $2.7 \%$ absolute systematic uncertainty on $\mathcal{B}\left(B^{-} \rightarrow D^{0} X\right)$ reflects the quadratic sum of $1.3 \%$ attributed to the track-finding efficiency, $1.6 \%$ to the description of the $m_{\mathrm{ES}}$ distribution by the $\Gamma_{A R G}$ and $\Gamma_{C B}$ functions, $0.8 \%$ to the description of the $m_{C(\bar{C})}$ signal distribution by the $\rho_{S}$ function, $1.4 \%$ to the particle identification, $0.5 \%$ to the Monte Carlo statistics, $0.3 \%$ to $c_{0}$, and $0.1 \%$ to $\mathcal{B}_{1}^{0}$.

The uncertainty affecting the track-finding efficiency is estimated with two different methods. The first uses a large inclusive sample of tracks with a minimum number of hits in the silicon vertex detector. The second relies on an $e^{+} e^{-} \rightarrow \tau^{+} \tau^{-}$control sample. From these, we derive a relative systematic uncertainty of $0.8 \%$ per track. 
TABLE III: Charm and anti-charm efficiency-corrected signal yields and fractional production rates. The uncertainties are statistical only.

\begin{tabular}{crrrrrrrr}
\hline \hline$C$ decay mode & \multicolumn{2}{c}{$C$ in recoil of $B_{\text {rec'd }}^{+} \bar{C}$ in recoil of $B_{\text {rec'd }}^{+}$} & \multicolumn{2}{c}{$C$ in recoil of $B_{\text {rec'd }}^{0}$} & \multicolumn{2}{c}{$\bar{C}$ in recoil of $B_{\text {rec'd }}^{0}$} \\
& $N^{-}(C \rightarrow f)$ & $\mathcal{B}_{c}^{-}(\%)$ & $N^{-}(\bar{C} \rightarrow \bar{f})$ & $\overline{\mathcal{B}}_{c}^{-}(\%)$ & $N^{0}(C \rightarrow f)$ & $\mathcal{B}_{c}^{0}(\%)$ & $N^{0}(\bar{C} \rightarrow \bar{f})$ & $\overline{\mathcal{B}}_{c}^{0}(\%)$ \\
\hline$D^{0} \rightarrow K^{-} \pi^{+}$ & $5898 \pm 126$ & $77.5 \pm 1.6$ & $691 \pm 52$ & $9.1 \pm 0.7$ & $1731 \pm 70$ & $41.1 \pm 1.7$ & $669 \pm 44$ & $15.9 \pm 1.0$ \\
$\rightarrow K^{-} \pi^{+} \pi^{-} \pi^{+}$ & $11010 \pm 383$ & $73.4 \pm 2.6$ & $1378 \pm 214$ & $9.2 \pm 1.4$ & $3418 \pm 239$ & $41.2 \pm 2.9$ & $1065 \pm 159$ & $12.8 \pm 1.9$ \\
\hline$D^{+} \rightarrow K^{-} \pi^{+} \pi^{+}$ & $1970 \pm 131$ & $10.8 \pm 0.7$ & $513 \pm 89$ & $2.8 \pm 0.5$ & $3044 \pm 122$ & $30.2 \pm 1.2$ & $869 \pm 74$ & $8.6 \pm 0.7$ \\
\hline$D_{s}^{+} \rightarrow \phi \pi^{+}$ & $85 \pm 24$ & $1.8 \pm 0.5$ & $385 \pm 42$ & $8.1 \pm 0.9$ & $97 \pm 21$ & $3.7 \pm 0.8$ & $227 \pm 30$ & $8.7 \pm 1.2$ \\
$\rightarrow \bar{K}^{* 0} K^{+}$ & $78 \pm 39$ & $1.3 \pm 0.6$ & $567 \pm 72$ & $9.3 \pm 1.2$ & $78 \pm 28$ & $2.3 \pm 0.8$ & $306 \pm 50$ & $9.1 \pm 1.5$ \\
$\rightarrow K_{S}^{0} K^{+}$ & $0 \pm 16$ & $0.0 \pm 0.5$ & $212 \pm 39$ & $6.6 \pm 1.2$ & $48 \pm 19$ & $2.7 \pm 1.1$ & $148 \pm 29$ & $8.3 \pm 1.6$ \\
\hline$\Lambda_{c}^{+} \rightarrow p K^{-} \pi^{+}$ & $288 \pm 52$ & $2.9 \pm 0.5$ & $210 \pm 45$ & $2.1 \pm 0.5$ & $240 \pm 41$ & $4.3 \pm 0.7$ & $124 \pm 30$ & $2.2 \pm 0.5$ \\
\hline \hline
\end{tabular}

TABLE IV: $\bar{B}$ branching fractions. The first uncertainty is statistical, the second is systematic, and the third reflects charm branching-fraction uncertainties [21, 23].

\begin{tabular}{ccccc}
\hline \hline & \multicolumn{2}{c}{ Correlated } & \multicolumn{2}{c}{ Anticorrelated } \\
$C$ & $\mathcal{B}\left(B^{-} \rightarrow C X\right)(\%)$ & $\mathcal{B}\left(\bar{B}^{0} \rightarrow C X\right)(\%)$ & $\mathcal{B}\left(B^{-} \rightarrow \bar{C} X\right)(\%)$ & $\mathcal{B}\left(\bar{B}^{0} \rightarrow \bar{C} X\right)(\%)$ \\
\hline$D^{0}$ & $78.6 \pm 1.6 \pm 2.7_{-1.9}^{+2.0}$ & $47.4 \pm 2.0 \pm 1.5_{-1.2}^{+1.3}$ & $8.6 \pm 0.6 \pm 0.3_{-0.2}^{+0.2}$ & $8.1 \pm 1.4 \pm 0.5_{-0.2}^{+0.2}$ \\
$D^{+}$ & $9.9 \pm 0.8 \pm 0.5_{-0.7}^{+0.8}$ & $36.9 \pm 1.6 \pm 1.4_{-2.3}^{+2.6}$ & $2.5 \pm 0.5 \pm 0.1_{-0.2}^{+0.2}$ & $2.3 \pm 1.1 \pm 0.3_{-0.1}^{+0.2}$ \\
& & & & $<3.9$ at $90 \% \mathrm{CL}$ \\
$D_{s}^{+}$ & $1.1_{-0.3}^{+0.4} \pm 0.1_{-0.1}^{+0.2}$ & $1.5 \pm 0.8 \pm 0.1_{-0.2}^{+0.2}$ & $7.9 \pm 0.6 \pm 0.4_{-1.0}^{+1.3}$ & $10.3 \pm 1.2 \pm 0.4_{-1.3}^{+1.7}$ \\
& & $<2.6$ at $90 \% \mathrm{CL}$ & & \\
$\Lambda_{c}^{+}$ & $2.8 \pm 0.5 \pm 0.3_{-0.6}^{+1.0}$ & $5.0 \pm 1.0 \pm 0.5_{-1.0}^{+1.8}$ & $2.1 \pm 0.5 \pm 0.2_{-0.4}^{+0.8}$ & $1.6 \pm 0.9 \pm 0.2_{-0.3}^{+0.6}$ \\
& & & & $<3.1$ at $90 \% \mathrm{CL}$ \\
\hline \hline
\end{tabular}

TABLE V: Fraction of anticorrelated charm as defined in Eq. (7).

\begin{tabular}{lcc}
\hline \hline Mode & $B^{-}$decays & $\bar{B}^{0}$ decays \\
\hline $\bar{D}^{0} X$ & $0.098 \pm 0.007 \pm 0.001$ & $0.146 \pm 0.022 \pm 0.006$ \\
$D^{-} X$ & $0.204 \pm 0.035 \pm 0.001$ & $0.058 \pm 0.028 \pm 0.006$ \\
& & $<0.098$ at $90 \% \mathrm{CL}$ \\
$D_{s}^{-} X$ & $0.884 \pm 0.038 \pm 0.002$ & $0.879 \pm 0.066 \pm 0.005$ \\
& & $>0.791$ at $90 \% \mathrm{CL}$ \\
$\bar{\Lambda}_{c}^{-} X$ & $0.427 \pm 0.071 \pm 0.001$ & $0.243_{-0.121}^{+0.119} \pm 0.003$ \\
& & $<0.403$ at $90 \% \mathrm{CL}$ \\
\hline \hline
\end{tabular}

The modeling of the $m_{\mathrm{ES}}$ distribution by the $\Gamma_{C B}$ and the $\Gamma_{A R G}$ functions affects both the charm signal yields and the numbers of reconstructed $B$ mesons used in normalizing the branching fractions. The corresponding uncertainty is dominated by the dependence of the $\Gamma_{A R G}$ shape parameter on the lower edge of the $m_{\mathrm{ES}}$ fit range. Varying the latter from 5.195 to $5.225 \mathrm{GeV} / c^{2}$ yields a variation in the branching fraction that is taken as systematic uncertainty. This range was chosen such that the branching fractions measured in the simulation change by \pm 1 standard deviation.

The uncertainty associated with the description of the charm signal mass shape by the $\rho_{S}$ function translates into an uncertainty on the charm reconstruction efficiency. It is estimated by fitting the simulated charm signal with a double instead of a single Gaussian.

The systematic uncertainties affecting the proton and charged kaon particle-identification efficiency are estimated using $D^{0} \rightarrow K^{-} \pi^{+}$and $\Lambda^{0} \rightarrow p \pi^{-}$samples recoiling against reconstructed $B^{+}$and $B^{0}$ mesons. The $D^{0}$ or $\Lambda^{0}$ signal yields are extracted in a manner similar to that described in Sec. IV A, both with and without applying the proton or kaon particle-identification requirements. The ratio of these yields on real and simulated samples is proportional to the particle-identification efficiency in the data and the simulation, respectively. The difference between these two efficiencies is then taken as an estimate of the corresponding the systematic uncertainty $(1.7 \%$ relative uncertainty per kaon and $1.3 \%$ per proton).

The statistical and systematic uncertainties in Table IV and Table V are computed separately for each charm decay mode; correlated errors are taken into account when averaging over $D^{0}$ and $D_{s}$ final states.

\section{Average charm production in $\bar{B}$ decays}

To extract $N_{c}$ from the results of Table IV, we still need to evaluate the $\bar{B} \rightarrow \Xi_{c} X$ and $\bar{B} \rightarrow(c \bar{c}) X$ branching fractions. Because there exists no absolute mea- 
surement of the $\Xi_{c}$-decay branching fraction, the absolute rates for correlated $\Xi_{c}$ production in $B$ decays are unknown $[14,25]$. Therefore, following the discussion in Sec. I, we assume that $\mathcal{B}\left(\bar{B} \rightarrow \Xi_{c} X\right)=\mathcal{B}(\bar{B} \rightarrow$ $\left.\bar{\Lambda}_{c}^{-} X\right)-\mathcal{B}\left(\bar{B} \rightarrow \Lambda_{c}^{+} \bar{\Lambda}_{c}^{-} \bar{K}(\pi)\right)[26]$. A recent measurement [27] indicates that $\bar{B} \rightarrow \Lambda_{c}^{+} \bar{\Lambda}_{c}^{-} \bar{K}$ decays have a branching fraction of the order of $7 \times 10^{-4}$, and thus can be neglected by comparison to $N_{c}^{-/ 0}$ (see also [2]). We take $\mathcal{B}(\bar{B} \rightarrow(c \bar{c}) X)=(2.3 \pm 0.3) \%$ [28, 29] and, using Eqs. (1) and (2), we obtain for charm production in $B^{-}$ decays:

$$
\begin{aligned}
N_{c}^{-} & =0.968 \pm 0.019 \pm 0.032_{-0.022}^{+0.026} \\
N_{\bar{c}}^{-} & =0.234 \pm 0.012 \pm 0.008_{-0.012}^{+0.016} \\
n_{c}^{-} & =1.202 \pm 0.023 \pm 0.040_{-0.029}^{+0.035}
\end{aligned}
$$

and in $\bar{B}^{0}$ decays :

$$
\begin{aligned}
N_{c}^{0} & =0.947 \pm 0.030 \pm 0.028_{-0.028}^{+0.035}, \\
N_{\bar{c}}^{0} & =0.246 \pm 0.024 \pm 0.009_{-0.014}^{+0.019}, \\
n_{c}^{0} & =1.193 \pm 0.030 \pm 0.034_{-0.035}^{+0.044} .
\end{aligned}
$$

The results reported here are consistent [30] with, and supersede those of Ref. [2]. The three-fold increase in integrated luminosity accounts for the substantial reduction in statistical error. The experimental systematic uncertainties have been similarly reduced, primarily through the use of the two-dimensional $\left[m_{\mathrm{ES}}, m_{C(\bar{C})}\right]$ fit, which takes correctly into account the correlation between the fitted number of reconstructed $B$ mesons and the corresponding charm yield.

\section{Isospin analysis}

The main source of anticorrelated $\bar{D}$ mesons produced in $\bar{B}$ decays is $b \rightarrow c \bar{c} s$ transitions. In these processes isospin should be conserved, leading to the expectation that : $\Gamma\left(B^{-} \rightarrow \bar{D}^{0} X\right)=\Gamma\left(\bar{B}^{0} \rightarrow D^{-} X\right)$ and $\Gamma\left(B^{-} \rightarrow\right.$ $\left.D^{-} X\right)=\Gamma\left(\bar{B}^{0} \rightarrow \bar{D}^{0} X\right)$. However, $\bar{D}$ mesons can also arise from $\bar{D}^{*}$ mesons, whose decay does not conserve isospin since the $\bar{D}^{* 0} \rightarrow D^{-} \pi^{+}$channel is kinematically forbidden. Thus isospin invariance actually requires :

$$
\begin{aligned}
& \Gamma_{d i r}\left(B^{-} \rightarrow \bar{D}^{0} X\right)=\Gamma_{d i r}\left(\bar{B}^{0} \rightarrow D^{-} X\right) \\
& \Gamma_{\text {dir }}\left(B^{-} \rightarrow D^{-} X\right)=\Gamma_{\text {dir }}\left(\bar{B}^{0} \rightarrow \bar{D}^{0} X\right) \\
& \Gamma\left(B^{-} \rightarrow \bar{D}^{* 0} X\right) \quad=\Gamma\left(\bar{B}^{0} \rightarrow D^{*-} X\right) \\
& \Gamma\left(B^{-} \rightarrow D^{*-} X\right) \quad=\Gamma\left(\bar{B}^{0} \rightarrow \bar{D}^{* 0} X\right)
\end{aligned}
$$

where $\Gamma_{d i r}(\bar{B} \rightarrow \bar{D} X)$ refers to the partial width of $\bar{B}$ meson decays to $\bar{D}$ mesons where the $\bar{D}$ state is not reached through a $\bar{D}^{*}$ cascade decay. Eqs. (8) lead to the following relations involving the measured anticorrelated $\bar{D}$ branching fractions in Table IV :

$$
\begin{aligned}
& r x^{*}=\mathcal{B}\left(B^{-} \rightarrow \bar{D}^{0} X\right)-\mathcal{B}\left(\bar{B}^{0} \rightarrow D^{-} X\right) \frac{\tau_{B^{+}}}{\tau_{B^{0}}} \\
& r x^{*}=\mathcal{B}\left(\bar{B}^{0} \rightarrow \bar{D}^{0} X\right) \frac{\tau_{B^{+}}}{\tau_{B^{0}}}-\mathcal{B}\left(B^{-} \rightarrow D^{-} X\right)
\end{aligned}
$$

and :

$$
\begin{aligned}
& x+x^{*}=\frac{1}{2}\left[\mathcal{B}\left(B^{-} \rightarrow \bar{D}^{0} X\right)+\mathcal{B}\left(B^{-} \rightarrow D^{-} X\right)\right. \\
& \left.+\mathcal{B}\left(\bar{B}^{0} \rightarrow \bar{D}^{0} X\right) \frac{\tau_{B}+}{\tau_{B^{0}}}+\mathcal{B}\left(\bar{B}^{0} \rightarrow D^{-} X\right) \frac{\tau_{B}+}{\tau_{B^{0}}}\right]
\end{aligned}
$$

where $\tau_{B}^{+} / \tau_{B}^{0}$ is the ratio of the $B^{+}$to the $B^{0}$ lifetime, $r=\mathcal{B}\left(D^{*-} \rightarrow \bar{D}^{0} \pi^{-}\right), x=\mathcal{B}_{\operatorname{dir}}\left(B^{-} \rightarrow \bar{D}^{0}+D^{-} X\right)$ and $x^{*}=\mathcal{B}\left(B^{-} \rightarrow \bar{D}^{* 0}+D^{*-} X\right)$ [31]. That both Eqs. (9) and (10) must be satisfied is a consequence of isospin invariance. From these two equations, we extract $x^{*}$ with a chi-squared method, and using in addition Eq. (11) we calculate :

$$
\begin{aligned}
\mathcal{B}\left(B^{-} \rightarrow \bar{D}^{* 0}+D^{*-} X\right) & =9.1 \pm 1.5 \pm 0.6 \% \\
\mathcal{B}_{\text {dir }}\left(B^{-} \rightarrow \bar{D}^{0}+D^{-} X\right) & =2.1 \pm 1.7 \pm 0.7 \% \\
& <4.5 \% \text { at } 90 \% \mathrm{CL} \\
\frac{\mathcal{B}_{\text {dir }}\left(\bar{B} \rightarrow \bar{D}^{0}+D^{-} X\right)}{\mathcal{B}\left(\bar{B} \rightarrow \bar{D}^{* 0}+D^{*-} X\right)} & =0.23_{-0.19}^{+0.25} \pm 0.09 \\
& <0.60 \text { at } 90 \% \mathrm{CL}
\end{aligned}
$$

Here the first uncertainty is statistical, the second is systematic and includes charm branching-fraction uncertainties, as well as those affecting the values of $\tau_{B}^{+} / \tau_{B}^{0}$ and $\mathcal{B}\left(D^{*-} \rightarrow \bar{D}^{0} \pi^{-}\right)$taken from Ref. [21]. The $\chi^{2}$ of the fit to Eqs. (9) and (10) is 0.01 for 1 degree of freedom.

\section{CHARM MOMENTUM DISTRIBUTIONS IN THE $\bar{B}$ REST FRAME}

As the four-momentum of the recoiling $\bar{B}$ is fully determined, each reconstructed charm hadron can be boosted into the rest frame of its parent $\bar{B}$, yielding the $p^{*}$ distribution of the corresponding (anti-charm) charm species in the $\bar{B}$ frame. The number of $C(\bar{C})$ candidates, their fractional production rates and the $\bar{B} \rightarrow C(\bar{C}) X$ branching fractions are then determined in each $p^{*}$ bin by the same methods as in Sec. IV, separately for $B^{-}$and $\bar{B}^{0}$ decays. The systematic uncertainties are assumed to be independent of $p^{*}$, except for the error associated with the $B^{0}\left(B^{+}\right)$contamination in the $B^{+}\left(B^{0}\right)$ sample : the latter is computed bin-by-bin with a relative uncertainty on $c_{+}$and $c_{0}$ increased to $100 \%$.

Figs. 6 and 7 show the result for correlated and anticorrelated $D^{0}, D^{+}, D_{s}$ and $\Lambda_{c}^{+}$production in $B^{-}$and $\bar{B}^{0}$ decays, respectively. The numerical values are tabulated in the Appendix.

Correlated $D^{0}$ and $D^{+}$(Figs. 6a, c and 7a, c) are produced in several types of transitions : $b \rightarrow c \ell^{-} \nu, b \rightarrow c \bar{u} d$ and $b \rightarrow c \bar{c} s$ which explains the fairly large spread of their momentum. High- $p^{*}$ correlated $D$ 's are produced in two-body decays such as $B^{-} \rightarrow D^{0} \pi^{-}$while low momentum $D$ 's might come from higher multiplicity final states such as $\bar{B} \rightarrow D \bar{D} K\left(X_{\text {light }}\right)$ where $X_{\text {light }}$ is any number of pions and/or photons. The latter processes are also the main source of anticorrelated $\bar{D}^{0}$ and $D^{-}$ 

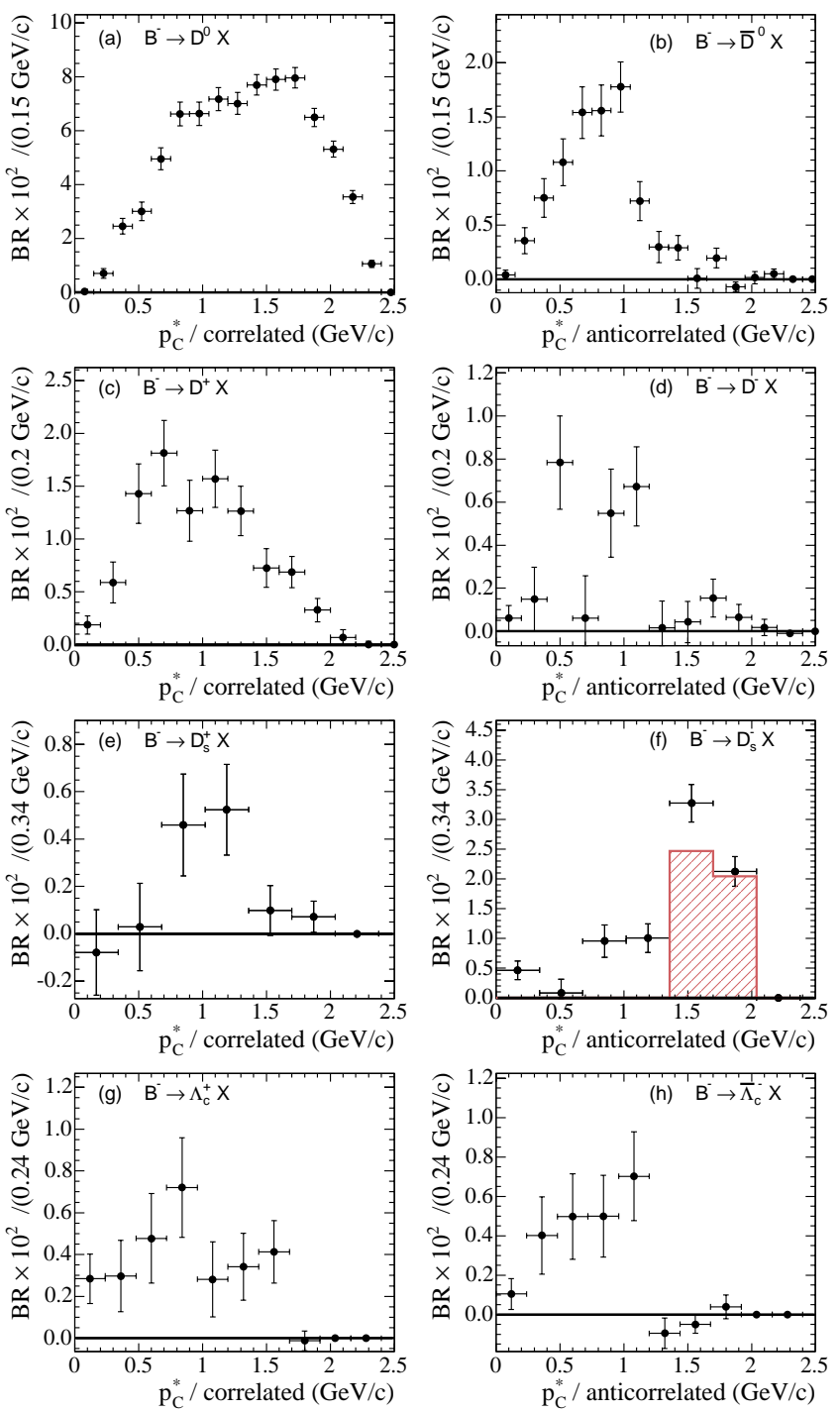

FIG. 6: Momentum spectra, in the $B^{-}$rest frame, of correlated (left) and anticorrelated (right) charm particles : $D^{0} / \bar{D}^{0}$ (a)(b), $D^{ \pm}(\mathrm{c})(\mathrm{d}), D_{s}^{ \pm}(\mathrm{e})(\mathrm{f}), \Lambda_{c}^{ \pm}(\mathrm{g})(\mathrm{h})$. The error bars are statistical only. The histogram in frame (f) represents the contribution of $B^{-} \rightarrow D^{(*) 0} D_{s}^{(*)-}$ two-body decays assuming the branching fractions of Ref. [21] and [23].

production (Figs. 6b, d and 7b, d) which explains why anticorrelated $\bar{D}$ spectra are softer than their correlated counterparts.

Anticorrelated $D_{s}^{-}$spectra (Figs. 6f and 7f) have a very different shape compared to anticorrelated $\bar{D}$ spectra. They are peaked at high $p^{*}$ values which is suggestive of the two-body decays $\bar{B} \rightarrow D^{(*)} D_{s}^{-}$and $\bar{B} \rightarrow D^{(*)} D_{s}^{*-}$. These decays represent a large fraction of the total anticorrelated $D_{s}^{-}$production as shown in Fig. 6. In contrast, the corresponding two-body processes $\bar{B} \rightarrow D^{(*)} D^{-}$and $\bar{B} \rightarrow D^{(*)} D^{*-}$ are Cabibbo-suppressed.

In the case of anticorrelated $\bar{\Lambda}_{c}^{-}$production associated with $\Xi_{c}$ production, for decays such as $\bar{B} \rightarrow$
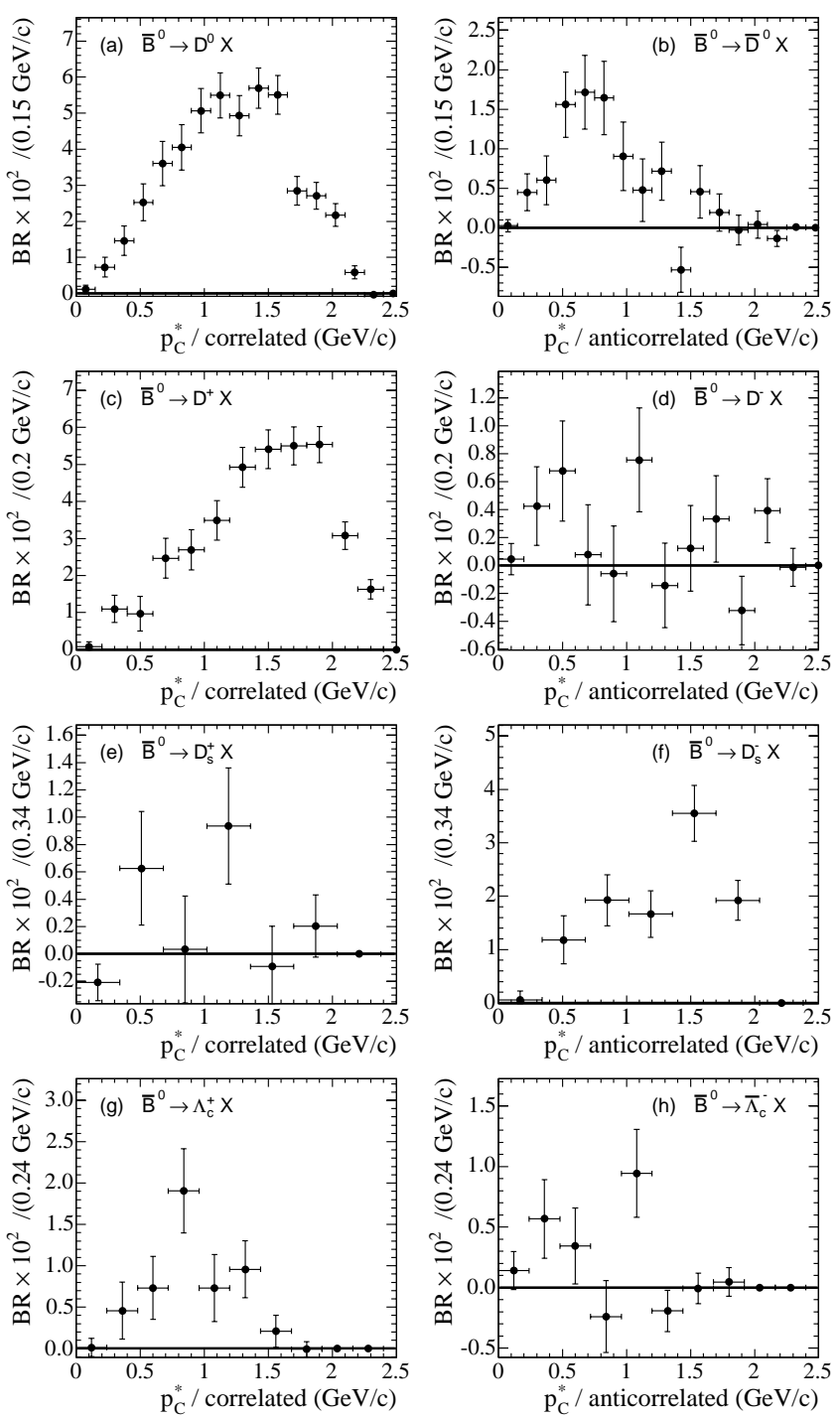

FIG. 7: Momentum spectra, in the $\bar{B}^{0}$ rest frame, of correlated (left) and anticorrelated (right) charm particles : $D^{0} / \bar{D}^{0}$ (a)(b), $D^{ \pm}$(c)(d), $D_{s}^{ \pm}$(e)(f), $\Lambda_{c}^{ \pm}(\mathrm{g})(\mathrm{h})$. The error bars are statistical only.

$\Xi_{c} \bar{\Lambda}_{c}^{-}\left(X_{\text {light }}\right)$, the anticorrelated $\bar{\Lambda}_{c}^{-}$spectra should have a cut-off at $p^{*}<1.15 \mathrm{GeV} / c$. This is actually observed in the data, both in $B^{-}$(Fig. 6h) and in $\bar{B}^{0}$ (Fig. 7h) decays.

\section{CONCLUSIONS}

We have measured the branching fractions for inclusive decays of $B$ mesons to flavor-tagged $D, D_{s}$ and $\Lambda_{c}^{+}$, separately for $B^{-}$and $\bar{B}^{0}$. We observe a significant production of anticorrelated $D^{0}$ and $D^{+}$mesons in $B$ decays, with the branching fractions reported in Table IV. These results are consistent with and supersede our previous measurement [2]. We find evidence for correlated 
$D_{s}^{+}$production in $B^{-}$decays, a process which has not been previously reported.

The sum of all correlated charm branching fractions, $N_{c}$, is compatible with 1 , for charged as well as for neutral $B$ mesons. The numbers of charm particles per $B^{-}$decay $\left(n_{c}^{-}=1.202 \pm 0.023 \pm 0.040_{-0.029}^{+0.035}\right)$ and per $\bar{B}^{0}$ decay $\left(n_{c}^{0}=1.193 \pm 0.030 \pm 0.034_{-0.035}^{+0.044}\right)$ are consistent with previous measurements $[2,4,28]$ and with theoretical expectations [8-11].

Assuming isospin conservation in the $b \rightarrow c \bar{c} s$ transition, we show that anticorrelated $\bar{D}$ mesons are mainly produced by cascade decays $\bar{B} \rightarrow \bar{D}^{*} X \rightarrow \bar{D} X$.

Finally, the technique developed for this analysis allows us to measure the inclusive momentum spectra of flavor-tagged $D, D_{s}$ and $\Lambda_{c}^{+}$in the rest frame of the $\bar{B}$ parent, separately in $B^{-}$and $\bar{B}^{0}$ decays, eventually providing insight into $B$-decay mechanisms.

\section{Acknowledgments}

We are grateful for the excellent luminosity and machine conditions provided by our PEP-II colleagues, and for the substantial dedicated effort from the computing organizations that support BABAR. The collaborating institutions wish to thank SLAC for its support and kind hospitality. This work is supported by DOE and NSF (USA), NSERC (Canada), IHEP (China), CEA and CNRS-IN2P3 (France), BMBF and DFG (Germany), INFN (Italy), FOM (The Netherlands), NFR (Norway), MIST (Russia), and PPARC (United Kingdom). Individuals have received support from CONACyT (Mexico), Marie Curie EIF (European Union), the A. P. Sloan Foundation, the Research Corporation, and the Alexander von Humboldt Foundation.
[1] Charge conjugation is implied for all decay processes mentioned in this paper.

[2] BABAR Collaboration, B. Aubert et al., Phys. Rev. D 70, 091106(R) (2004).

[3] CLEO collaboration, L. Gibbons et al.,Phys. Rev. D 56, 3783 (1997).

[4] CLEO collaboration, T.E. Coan et al., Phys. Rev. Lett. 80, 1150 (1998).

[5] DELPHI collaboration, J. Abdallah et al., Phys. Lett. B 561, 26 (2003).

[6] CLEO collaboration, R. Ammar et al., Phys. Rev. D 55, 13 (1997).

[7] BELLE collaboration, R. Seuster et al., Phys. Rev. D 73, 032002 (2006).

[8] E. Bagan et al., Phys. Lett. B 351, 546 (1995).

[9] G. Buchalla et al., Phys. Lett. B 364, 188 (1995).

[10] M. Neubert, 17th Int. Symposium on Lepton-Photon Interactions, 10-15 Aug 95, Beijing, China, p. 298 (World Scientific).

[11] A. Lenz, hep-ph/0011258 and references therein.

[12] ALEPH collaboration, R. Barate et al., Eur. Phys. Jour. C 4, 387 (1998).

[13] BABAR collaboration, B. Aubert et al., Phys. Rev. D 68, 092001 (2003).

[14] BELLE collaboration, R. Chistov et al., hep-ex/0510074.

[15] C.W. Bauer, B. Grinstein, D. Pirjol, I.W. Stewart, Phys. Rev. D 67, 014010 (2003).

[16] I. Bigi, L. Maiani, F. Piccinini, A.D. Polosa, V. Riquer, Phys. Rev. D 72, 114016 (2005).

[17] BABAR Collaboration, B. Aubert et al., Nucl. Instr. Methods Phys. Res., Sect. A 479,1 (2002).

[18] GEANT4 Collaboration, S. Agostinelli et al. Nucl. Instr. Methods Phys. Res., Sect. A 506, 250 (2003).

[19] CRYSTAL BALL collaboration, T. Skwarnicki et al., DESY F31-86-02.

[20] ARGUS collaboration, H. Albrecht et al., Z. Phys. C 48, 543 (1990).

[21] Particle Data Group, S. Eidelman et al., Phys. Lett. B 592, 1 (2004).
[22] We consider any $K^{+} K^{-}$combination with an invariant mass in the range $1010.6<m_{K^{+} K^{-}}<1028.6 \mathrm{MeV} / \mathrm{c}^{2}$ to be a $\phi$ meson when reconstructing the $D_{s}^{+} \rightarrow \phi(\rightarrow$ $\left.K^{+} K^{-}\right) \pi^{+}$decay.

[23] BABAR collaboration, B. Aubert et al., Phys. Rev. D 71, 091104 (2005).

[24] In some cases, a satellite contribution needs to be added. It includes a reflection from $D^{0} \rightarrow K^{-} K^{+}$in the $D^{0} \rightarrow$ $K^{-} \pi^{+}$mass spectra and a signal at the $D^{+}$mass (from $D^{+} \rightarrow \phi \pi^{+}, \bar{K}^{* 0} K^{+}, K_{S}^{0} K^{+}$Cabibbo-suppressed decays) in the $D_{s}$ mass spectra.

[25] CLEO collaboration, B. Barish et al., Phys. Rev. Lett. 79, 3599 (1997).

[26] We also neglect the contribution of the $\bar{B} \rightarrow D \Lambda^{0} \bar{\Lambda}_{c}^{-}$ decays because of the very small phase space available.

[27] BELLE collaboration, N. Gabyshev et al., hepex/0508015.

[28] ALEPH, CDF, DELPHI, L3, OPAL, SLD combined results, hep-ex/0112028, CERN-EP/2001-050.

[29] M. Beneke et al., Phys. Rev. D 59, 054003 (1999).

[30] Provided one takes into account the $25 \%$ change in the $D_{s}^{+}$branching fraction [21, 23] assumed in interpreting the $D_{s}^{+} \rightarrow \phi \pi^{+}$yield.

[31] Assuming isospin conservation, we have also : $x=$ $\mathcal{B}_{\text {dir }}\left(\bar{B}^{0} \rightarrow \bar{D}^{0}+D^{-} X\right) \times \frac{\tau_{B}+}{\tau_{B^{0}}}$ and $x^{*}=\mathcal{B}\left(\bar{B}^{0} \rightarrow\right.$ $\left.\bar{D}^{* 0}+D^{*-} X\right) \times \frac{\tau_{B}+}{\tau_{B^{0}}}$

\section{APPENDIX : CHARM $p^{*}$ SPECTRA}

This appendix tabulates the measured $p^{*}$ dependence of the branching fractions displayed in Figs. 6 and 7 . In Tables VI to XIII, the first uncertainty is statistical, the second is systematic and includes charm branchingfraction uncertainties. Within each table, the statistical uncertainties are uncorrelated whereas the systematic errors are fully correlated. 
TABLE VI: Correlated and anticorrelated $D^{0}$ production in $B^{-}$decays.

\begin{tabular}{ccc}
\hline \hline & correlated prod. & anticorrelated prod. \\
$p^{*}$ range $(\mathrm{GeV} / c)$ & $\mathcal{B}\left(B^{-} \rightarrow X_{c} X\right)(\%)$ & $\mathcal{B}\left(B^{-} \rightarrow X_{\bar{c}} X\right)(\%)$ \\
\hline $0.00-0.15$ & $0.03 \pm 0.06 \pm 0.01$ & $0.04 \pm 0.04 \pm 0.01$ \\
$0.15-0.30$ & $0.70 \pm 0.18 \pm 0.03$ & $0.36 \pm 0.12 \pm 0.02$ \\
$0.30-0.45$ & $2.45 \pm 0.29 \pm 0.11$ & $0.75 \pm 0.18 \pm 0.03$ \\
$0.45-0.60$ & $3.01 \pm 0.34 \pm 0.13$ & $1.08 \pm 0.22 \pm 0.05$ \\
$0.60-0.75$ & $4.96 \pm 0.40 \pm 0.22$ & $1.54 \pm 0.24 \pm 0.07$ \\
$0.75-0.90$ & $6.62 \pm 0.44 \pm 0.30$ & $1.56 \pm 0.23 \pm 0.07$ \\
$0.90-1.05$ & $6.63 \pm 0.43 \pm 0.30$ & $1.78 \pm 0.23 \pm 0.07$ \\
$1.05-1.20$ & $7.18 \pm 0.43 \pm 0.32$ & $0.72 \pm 0.18 \pm 0.04$ \\
$1.20-1.35$ & $7.01 \pm 0.41 \pm 0.32$ & $0.30 \pm 0.14 \pm 0.05$ \\
$1.35-1.50$ & $7.70 \pm 0.38 \pm 0.35$ & $0.29 \pm 0.11 \pm 0.02$ \\
$1.50-1.65$ & $7.90 \pm 0.39 \pm 0.36$ & $0.01 \pm 0.09 \pm 0.05$ \\
$1.65-1.80$ & $7.96 \pm 0.38 \pm 0.40$ & $0.20 \pm 0.09 \pm 0.02$ \\
$1.80-1.95$ & $6.49 \pm 0.33 \pm 0.32$ & $-0.07 \pm 0.04 \pm 0.02$ \\
$1.95-2.10$ & $5.32 \pm 0.29 \pm 0.26$ & $0.02 \pm 0.06 \pm 0.02$ \\
$2.10-2.25$ & $3.54 \pm 0.24 \pm 0.19$ & $0.05 \pm 0.04 \pm 0.01$ \\
$2.25-2.40$ & $1.06 \pm 0.13 \pm 0.06$ & - \\
\hline
\end{tabular}

TABLE VII: Correlated and anticorrelated $D^{+}$production in $B^{-}$decays.

\begin{tabular}{ccc}
\hline \hline & correlated prod. & anticorrelated prod. \\
$p^{*}$ range $(\mathrm{GeV} / c)$ & $\mathcal{B}\left(B^{-} \rightarrow X_{c} X\right)(\%)$ & $\mathcal{B}\left(B^{-} \rightarrow X_{\bar{c}} X\right)(\%)$ \\
\hline $0.00-0.20$ & $0.19 \pm 0.09 \pm 0.02$ & $0.06 \pm 0.06 \pm 0.01$ \\
$0.20-0.40$ & $0.59 \pm 0.19 \pm 0.06$ & $0.15 \pm 0.15 \pm 0.02$ \\
$0.40-0.60$ & $1.43 \pm 0.28 \pm 0.14$ & $0.78 \pm 0.22 \pm 0.07$ \\
$0.60-0.80$ & $1.81 \pm 0.31 \pm 0.17$ & $0.06 \pm 0.20 \pm 0.02$ \\
$0.80-1.00$ & $1.27 \pm 0.29 \pm 0.13$ & $0.55 \pm 0.21 \pm 0.05$ \\
$1.00-1.20$ & $1.57 \pm 0.27 \pm 0.16$ & $0.67 \pm 0.18 \pm 0.06$ \\
$1.20-1.40$ & $1.27 \pm 0.23 \pm 0.16$ & $0.02 \pm 0.12 \pm 0.03$ \\
$1.40-1.60$ & $0.72 \pm 0.18 \pm 0.15$ & $0.04 \pm 0.10 \pm 0.04$ \\
$1.60-1.80$ & $0.69 \pm 0.15 \pm 0.16$ & $0.15 \pm 0.09 \pm 0.04$ \\
$1.80-2.00$ & $0.33 \pm 0.11 \pm 0.16$ & $0.06 \pm 0.06 \pm 0.03$ \\
$2.00-2.20$ & $0.07 \pm 0.07 \pm 0.09$ & $0.02 \pm 0.04 \pm 0.03$ \\
\hline \hline
\end{tabular}

TABLE VIII: Correlated and anticorrelated $D_{s}$ production in $B^{-}$decays.

\begin{tabular}{ccl}
\hline \hline & correlated prod. & anticorrelated prod. \\
$p^{*}$ range $(\mathrm{GeV} / c)$ & $\mathcal{B}\left(B^{-} \rightarrow X_{c} X\right)(\%)$ & $\mathcal{B}\left(B^{-} \rightarrow X_{\bar{c}} X\right)(\%)$ \\
\hline $0.00-0.34$ & $-0.08 \pm 0.18 \pm 0.02$ & $0.46 \pm 0.16 \pm 0.07$ \\
$0.34-0.68$ & $0.03 \pm 0.18 \pm 0.03$ & $0.08 \pm 0.23 \pm 0.04$ \\
$0.68-1.02$ & $0.46 \pm 0.22 \pm 0.09$ & $0.95 \pm 0.27 \pm 0.14$ \\
$1.02-1.36$ & $0.52 \pm 0.19 \pm 0.11$ & $1.00 \pm 0.24 \pm 0.15$ \\
$1.36-1.70$ & $0.10 \pm 0.11 \pm 0.03$ & $3.27 \pm 0.32 \pm 0.49$ \\
$1.70-2.04$ & $0.07 \pm 0.07 \pm 0.02$ & $2.13 \pm 0.25 \pm 0.32$ \\
\hline \hline
\end{tabular}

TABLE IX: Correlated and anticorrelated $\Lambda_{c}^{+}$production in $B^{-}$decays.

\begin{tabular}{ccc}
\hline \hline & correlated prod. & anticorrelated prod. \\
$p^{*}$ range $(\mathrm{GeV} / c)$ & $\mathcal{B}\left(B^{-} \rightarrow X_{c} X\right)(\%)$ & $\mathcal{B}\left(B^{-} \rightarrow X_{\bar{c}} X\right)(\%)$ \\
\hline $0.00-0.24$ & $0.28 \pm 0.12 \pm 0.09$ & $0.10 \pm 0.08 \pm 0.03$ \\
$0.24-0.48$ & $0.30 \pm 0.17 \pm 0.09$ & $0.40 \pm 0.20 \pm 0.12$ \\
$0.48-0.72$ & $0.48 \pm 0.21 \pm 0.15$ & $0.50 \pm 0.22 \pm 0.15$ \\
$0.72-0.96$ & $0.72 \pm 0.24 \pm 0.22$ & $0.50 \pm 0.21 \pm 0.15$ \\
$0.96-1.20$ & $0.28 \pm 0.18 \pm 0.09$ & $0.70 \pm 0.23 \pm 0.21$ \\
$1.20-1.44$ & $0.34 \pm 0.16 \pm 0.11$ & $-0.10 \pm 0.08 \pm 0.03$ \\
$1.44-1.68$ & $0.41 \pm 0.15 \pm 0.13$ & $-0.05 \pm 0.05 \pm 0.01$ \\
\hline \hline
\end{tabular}


TABLE X: Correlated and anticorrelated $D^{0}$ production in $\bar{B}^{0}$ decays.

\begin{tabular}{ccc}
\hline \hline & correlated prod. & anticorrelated prod. \\
$p^{*}$ range $(\mathrm{GeV} / c)$ & $\mathcal{B}\left(B^{-} \rightarrow X_{c} X\right)(\%)$ & $\mathcal{B}\left(B^{-} \rightarrow X_{\bar{c}} X\right)(\%)$ \\
\hline $0.00-0.15$ & $0.11 \pm 0.12 \pm 0.01$ & $0.03 \pm 0.08 \pm 0.01$ \\
$0.15-0.30$ & $0.73 \pm 0.28 \pm 0.03$ & $0.45 \pm 0.23 \pm 0.03$ \\
$0.30-0.45$ & $1.46 \pm 0.41 \pm 0.07$ & $0.60 \pm 0.31 \pm 0.04$ \\
$0.45-0.60$ & $2.53 \pm 0.51 \pm 0.11$ & $1.56 \pm 0.41 \pm 0.11$ \\
$0.60-0.75$ & $3.60 \pm 0.62 \pm 0.16$ & $1.71 \pm 0.47 \pm 0.12$ \\
$0.75-0.90$ & $4.05 \pm 0.63 \pm 0.20$ & $1.64 \pm 0.46 \pm 0.12$ \\
$0.90-1.05$ & $5.07 \pm 0.61 \pm 0.23$ & $0.90 \pm 0.43 \pm 0.07$ \\
$1.05-1.20$ & $5.50 \pm 0.62 \pm 0.25$ & $0.48 \pm 0.40 \pm 0.06$ \\
$1.20-1.35$ & $4.93 \pm 0.56 \pm 0.24$ & $0.72 \pm 0.37 \pm 0.08$ \\
$1.35-1.50$ & $5.70 \pm 0.56 \pm 0.27$ & $-0.53 \pm 0.29 \pm 0.07$ \\
$1.50-1.65$ & $5.51 \pm 0.53 \pm 0.27$ & $0.45 \pm 0.33 \pm 0.09$ \\
$1.65-1.80$ & $2.85 \pm 0.40 \pm 0.23$ & $0.19 \pm 0.24 \pm 0.07$ \\
$1.80-1.95$ & $2.71 \pm 0.37 \pm 0.19$ & $-0.03 \pm 0.19 \pm 0.06$ \\
$1.95-2.10$ & $2.17 \pm 0.32 \pm 0.16$ & $0.04 \pm 0.17 \pm 0.05$ \\
$2.10-2.25$ & $0.58 \pm 0.18 \pm 0.11$ & $-0.14 \pm 0.10 \pm 0.02$ \\
\hline \hline
\end{tabular}

TABLE XI: Correlated and anticorrelated $D^{+}$production in $\bar{B}^{0}$ decays.

\begin{tabular}{ccc}
\hline \hline & correlated prod. & anticorrelated prod. \\
$p^{*}$ range $(\mathrm{GeV} / c)$ & $\mathcal{B}\left(B^{-} \rightarrow X_{c} X\right)(\%)$ & $\mathcal{B}\left(B^{-} \rightarrow X_{\bar{c}} X\right)(\%)$ \\
\hline $0.00-0.20$ & $0.08 \pm 0.12 \pm 0.01$ & $0.05 \pm 0.11 \pm 0.01$ \\
$0.20-0.40$ & $1.10 \pm 0.37 \pm 0.09$ & $0.42 \pm 0.28 \pm 0.07$ \\
$0.40-0.60$ & $0.97 \pm 0.47 \pm 0.08$ & $0.68 \pm 0.36 \pm 0.11$ \\
$0.60-0.80$ & $2.47 \pm 0.54 \pm 0.19$ & $0.08 \pm 0.36 \pm 0.02$ \\
$0.80-1.00$ & $2.70 \pm 0.54 \pm 0.21$ & $-0.06 \pm 0.34 \pm 0.02$ \\
$1.00-1.20$ & $3.49 \pm 0.53 \pm 0.28$ & $0.76 \pm 0.37 \pm 0.12$ \\
$1.20-1.40$ & $4.92 \pm 0.54 \pm 0.39$ & $-0.14 \pm 0.30 \pm 0.04$ \\
$1.40-1.60$ & $5.41 \pm 0.52 \pm 0.44$ & $0.12 \pm 0.31 \pm 0.04$ \\
$1.60-1.80$ & $5.50 \pm 0.51 \pm 0.45$ & $0.33 \pm 0.31 \pm 0.06$ \\
$1.80-2.00$ & $5.54 \pm 0.49 \pm 0.45$ & $-0.32 \pm 0.25 \pm 0.06$ \\
$2.00-2.20$ & $3.08 \pm 0.37 \pm 0.25$ & $0.39 \pm 0.23 \pm 0.06$ \\
$2.20-2.40$ & $1.63 \pm 0.26 \pm 0.13$ & $-0.01 \pm 0.14 \pm 0.01$ \\
\hline \hline
\end{tabular}

TABLE XII: Correlated and anticorrelated $D_{s}$ production in $\bar{B}^{0}$ decays.

\begin{tabular}{ccc}
\hline \hline & correlated prod. & anticorrelated prod. \\
$p^{*}$ range $(\mathrm{GeV} / c)$ & $\mathcal{B}\left(B^{-} \rightarrow X_{c} X\right)(\%)$ & $\mathcal{B}\left(B^{-} \rightarrow X_{\bar{c}} X\right)(\%)$ \\
\hline $0.00-0.34$ & $-0.21 \pm 0.13 \pm 0.03$ & $0.06 \pm 0.16 \pm 0.02$ \\
$0.34-0.68$ & $0.63 \pm 0.42 \pm 0.09$ & $1.18 \pm 0.45 \pm 0.18$ \\
$0.68-1.02$ & $0.03 \pm 0.39 \pm 0.01$ & $1.92 \pm 0.48 \pm 0.29$ \\
$1.02-1.36$ & $0.94 \pm 0.43 \pm 0.14$ & $1.66 \pm 0.43 \pm 0.25$ \\
$1.36-1.70$ & $-0.09 \pm 0.29 \pm 0.03$ & $3.55 \pm 0.52 \pm 0.54$ \\
$1.70-2.04$ & $0.20 \pm 0.23 \pm 0.04$ & $1.92 \pm 0.37 \pm 0.29$ \\
\hline \hline
\end{tabular}

TABLE XIII: Correlated and anticorrelated $\Lambda_{c}^{+}$production in $\bar{B}^{0}$ decays.

\begin{tabular}{ccc}
\hline \hline & correlated prod. & anticorrelated prod. \\
$p^{*}$ range $(\mathrm{GeV} / c)$ & $\mathcal{B}\left(B^{-} \rightarrow X_{c} X\right)(\%)$ & $\mathcal{B}\left(B^{-} \rightarrow X_{\bar{c}} X\right)(\%)$ \\
\hline $0.00-0.24$ & $0.01 \pm 0.11 \pm 0.01$ & $0.14 \pm 0.16 \pm 0.05$ \\
$0.24-0.48$ & $0.46 \pm 0.34 \pm 0.15$ & $0.57 \pm 0.33 \pm 0.19$ \\
$0.48-0.72$ & $0.73 \pm 0.38 \pm 0.23$ & $0.34 \pm 0.31 \pm 0.12$ \\
$0.72-0.96$ & $1.90 \pm 0.51 \pm 0.60$ & $-0.24 \pm 0.30 \pm 0.08$ \\
$0.96-1.20$ & $0.73 \pm 0.40 \pm 0.23$ & $0.94 \pm 0.36 \pm 0.32$ \\
$1.20-1.44$ & $0.96 \pm 0.35 \pm 0.30$ & $-0.19 \pm 0.17 \pm 0.07$ \\
$1.44-1.68$ & $0.21 \pm 0.19 \pm 0.07$ & $-0.01 \pm 0.13 \pm 0.01$ \\
\hline \hline
\end{tabular}

